\title{
On Varifold Solutions of Two-Phase Incompressible Viscous Flow with Surface Tension
}

\author{
Karen Yeressian
}

Communicated by K. Pileckas

\begin{abstract}
In this paper using diffuse approximations the existence of a varifold solution to the two-phase Newtonian incompressible viscous flow problem is derived. On the free surface between the two phases we consider surface tension force. Also we prove that for axisymmetric, possibly with swirl, initial velocities and cylindrically symmetric initial volumes occupied by each fluid there exists a global in time axisymmetric, with swirl, solution.
\end{abstract}

Mathematics Subject Classification. Primary 35Q30, 76D27, 76D45, 76U05; Secondary 35Q35.

Keywords. Axisymmetric with swirl, free surface, incompressible viscous flow, surface-tension, two-phase flow, varifold solutions.

\section{Introduction}

The notion of varifold solution was introduced by Plotnikov [9] for a two-dimensional flow of shear thickening fluids. For a varifold solution the mean curvature appearing in the surface tension force is interpreted as the first variation of a general varifold. In [2] the existence of varifold and weak measure valued solutions for a large class of two-phase incompressible viscous flows is established. In this paper we obtain the existence of a varifold solution to the two-phase incompressible viscous flow problem in $\mathbb{R}^{3}$ with surface tension on the interface between the two fluids using rather simple diffuse approximations.

Although the uniqueness of the solution is not known, one expects that if the initial velocities and volumes occupied by the two fluids are axisymmetric with respect to the axis $e_{3}$, then there should exist an axisymmetric, with swirl, solution in all time intervals $(0, T)$. In this paper we also prove the existence of this axisymmetric, with swirl, solution as a varifold solution.

Many authors (cf. $[1,3,4,8,12-14,17])$ have worked on rather regular solutions of such free boundary problems. In the case of initial value problems by these results one has the well-posedness locally in time unless the initial state is close enough to equilibrium states. The approach by varifold solutions is a phase-field formulation and allows one to obtain global in time solutions with arbitrary initial states.

\subsection{Problem Setting}

By $\Gamma(t)$ we denote the free boundary and by $\Omega_{i}(t)$ for $i=0,1$ respectively the volumes occupied by the two phases. So we have $\mathbb{R}^{3}=\Omega_{0}(t) \cup \Gamma(t) \cup \Omega_{1}(t)$. By $u$ and $p$ we denote respectively the velocity field and the pressure in both phases. The two-phase Newtonian incompressible flow with surface tension force on the free interface is the following system of equations 


$$
\begin{cases}\partial_{t} u+\operatorname{div}(u \otimes u)=\operatorname{div}(S(p, D u)) & \text { in } \mathbb{R}^{3} \backslash \Gamma(t) \text { for } 0<t<T, \\ \operatorname{div}(u)=0 & \text { in } \mathbb{R}^{3} \backslash \Gamma(t) \text { for } 0<t<T, \\ {[S(p, D u)]_{\Gamma(t)} n_{\Gamma}=-\kappa_{\Gamma} n_{\Gamma}} & \text { on } \Gamma(t) \text { for } 0<t<T, \\ u \text { is continuous across } \Gamma(t), & \text { in } \mathbb{R}^{3}, \\ u(0)=u_{0} & \\ \text { Velocity of } \Gamma(t) \text { equals } u_{\Gamma(t)}^{\perp}, & \\ \Omega_{1}(0)=\Omega_{1,0} . & \end{cases}
$$

Here $S(p, D u)=D u-p I$ is the stress tensor, $2 D u=\nabla u+(\nabla u)^{T}$,

$$
\begin{aligned}
{[S(p, D u)]_{\Gamma(t)} } & =\text { jump of } S(p, D u) \text { across } \Gamma(t) \\
& =\lim _{y \rightarrow x, y \in \Omega_{1}} S(D u, p)-\lim _{y \rightarrow x, y \in \Omega_{0}} S(D u, p),
\end{aligned}
$$

$n_{\Gamma}$ is the outward with respect to $\Omega_{1}(t)$ normal on $\Gamma(t), \kappa_{\Gamma}=\operatorname{div}_{\Gamma}\left(n_{\Gamma}\right)$ is the scalar mean curvature of the interface $\Gamma$ with respect to $n_{\Gamma}, u_{0}$ is the initial velocity and $\Omega_{1,0} \subset \mathbb{R}^{3}$ is the initial volume occupied by the fluid with index 1 such that the initial area of the free boundary is finite, i.e. $|\Gamma(0)|=\left|\partial \Omega_{1,0}\right|<\infty$.

\subsection{Weak Formulation Assuming Smoothness}

To describe the notion of varifold solution let us first consider the weak formulation in the case of classical solutions.

By multiplying the momentum equation in (1.1) by $\varphi \in\left(C_{c}^{\infty}\left((-\infty, T) \times \mathbb{R}^{3}\right)\right)^{3}$ with $\operatorname{div}(\varphi)=0$ and partial integrations we obtain

$$
\begin{aligned}
& -\int_{\mathbb{R}^{3}} u_{0}^{T} \varphi(0) d x-\int_{0}^{T} \int_{\mathbb{R}^{3}}\left(u^{T} \partial_{t} \varphi+(u \otimes u): \nabla \varphi\right) d x d t+\int_{0}^{T} \int_{\mathbb{R}^{3}} D u: D \varphi d x d t \\
& =-\int_{0}^{T} \int_{\Gamma} \kappa_{\Gamma} \varphi \cdot n_{\Gamma} \mathcal{H}^{2}(d x) d t
\end{aligned}
$$

here $\mathcal{H}^{2}$ denotes the two dimensional Hausdorff measure on $\mathbb{R}^{3}$, with the initial value $u(0)=u_{0}$.

We denote by $\varphi^{\perp}=\left(\varphi \cdot n_{\Gamma}\right) n_{\Gamma}$ the normal component of $\varphi$ on $\Gamma$ and $\operatorname{by}_{\operatorname{proj}_{\Gamma}}(\varphi)=\varphi-\varphi^{\perp}$ then projection of $\varphi$ on the tangent plane (at the point $x \in \Gamma$ ) of $\Gamma$. Because $\Gamma$ as a manifold has no boundary, by the divergence theorem we have that

$$
\int_{\Gamma} \operatorname{div}_{\Gamma}\left(\operatorname{proj}_{\Gamma}(\varphi)\right) \mathcal{H}^{2}(d x)=0
$$

Also it is known (cf. [11]) that denoting by $H_{\Gamma}=-\kappa_{\Gamma} n_{\Gamma}$ the vector valued mean curvature of $\Gamma$ we have

$$
\operatorname{div}_{\Gamma}\left(\varphi^{\perp}\right)=-\varphi \cdot H_{\Gamma}
$$

Using (1.3) and (1.4) we compute

$$
\begin{aligned}
\int_{\Gamma} \kappa_{\Gamma} \varphi \cdot n_{\Gamma} \mathcal{H}^{2}(d x) & =-\int_{\Gamma} \varphi \cdot H_{\Gamma} \mathcal{H}^{2}(d x)=\int_{\Gamma} \operatorname{div}_{\Gamma}\left(\varphi^{\perp}\right) \mathcal{H}^{2}(d x) \\
& =\int_{\Gamma} \operatorname{div}_{\Gamma}(\varphi) \mathcal{H}^{2}(d x)=\int_{\Gamma}\left(\operatorname{div}(\varphi)-n_{\Gamma} \cdot \partial_{n_{\Gamma}} \varphi\right) \mathcal{H}^{2}(d x) \\
& =\int_{\Gamma}\left(I-n_{\Gamma} \otimes n_{\Gamma}\right): \nabla \varphi \mathcal{H}^{2}(d x) .
\end{aligned}
$$


Thus by (1.2) and (1.5) we have

$$
\begin{aligned}
& -\int_{\mathbb{R}^{3}} u_{0}^{T} \varphi(0) d x-\int_{0}^{T} \int_{\mathbb{R}^{3}}\left(u^{T} \partial_{t} \varphi+(u \otimes u): \nabla \varphi\right) d x d t+\int_{0}^{T} \int_{\mathbb{R}^{3}} D u: D \varphi d x d t \\
& \quad=-\int_{0}^{T} \int_{\Gamma}\left(I-n_{\Gamma} \otimes n_{\Gamma}\right): \nabla \varphi \mathcal{H}^{2}(d x) d t .
\end{aligned}
$$

Let $\chi=1_{\Omega_{1}(t)}(x)$ then by the last two lines of $(1.1), \chi$ satisfies

$$
\left\{\begin{array}{l}
\partial_{t} \chi+u \cdot \nabla \chi=0 \quad \text { in }(0, T) \times \mathbb{R}^{3} \\
\chi(0)=\chi_{0}
\end{array}\right.
$$

where $\chi_{0}=1_{\Omega_{1,0}}$.

\subsection{Varifold Solution}

In the case of smooth $\Gamma$ we can write the integral on $\Gamma$ appearing on the right hand side of the Eq. (1.6) as follows

$$
\int_{\Gamma(t)}\left(I-n_{\Gamma(t)} \otimes n_{\Gamma(t)}\right): \nabla \varphi \mathcal{H}^{2}(d x)=\int_{\mathbb{R}^{3} \times \mathbb{S}^{2}}(I-y \otimes y): \nabla \varphi V(t)(d(x, y))
$$

where $V(t) \in \mathcal{M}\left(\mathbb{R}^{3} \times \mathbb{S}^{2}\right)$ is defined by

$$
V(t)(A \times B)=\int_{A \cap \Gamma(t)} \delta_{n_{\Gamma(t)}(x)}(B) \mathcal{H}^{2}(d x) \quad \text { for } A \in \mathcal{B}\left(\mathbb{R}^{3}\right) \text { and } B \in \mathcal{B}\left(\mathbb{S}^{2}\right)
$$

here $\mathcal{B}(X)$ denotes the Borel $\sigma$-algebra of $X$.

For each $t$ the measure $V(t)$ describes the surface $\Gamma(t)$ together with its normal in a weak measure theoretic sense. For our purpose, by saying a general 2-varifold in $\mathbb{R}^{3}$ we understand a bounded nonnegative Radon measure on $\mathbb{R}^{3} \times \mathbb{S}^{2}$. Thus $V(t)$ is the general 2-varifold associated with the smooth surface $\Gamma(t)$. For the theory of general varifolds one may refer to [11].

We are not able to prove the existence of solutions with smooth enough interface, but we are able to prove the existence of a time dependent 2-varifold $V(t)$ describing $\Gamma(t)$. We shall show that there exists

$$
V \in L_{w^{*}}^{\infty}\left(0, T, \mathcal{M}\left(\mathbb{R}^{3} \times \mathbb{S}^{2}\right)\right)
$$

such that the term on the right hand side of (1.6) is replaced by the (time integral of the) right hand side of (1.7). The space $L_{w^{*}}^{\infty}\left(0, T, \mathcal{M}\left(\mathbb{R}^{3} \times \mathbb{S}^{2}\right)\right)$ is defined in Sect. 2 .

For a 2 -varifold $\mathcal{V} \in \mathcal{M}\left(\mathbb{R}^{3} \times \mathbb{S}^{2}\right)$ the first variation functional $\delta \mathcal{V}$ is defined by

$$
\langle\delta \mathcal{V}, \varphi\rangle=\int_{\mathbb{R}^{3} \times \mathbb{S}^{2}}(I-y \otimes y): \nabla \varphi(x) \mathcal{V}(d(x, y)) \quad \text { for } \varphi \in\left(C_{0}^{1}\left(\mathbb{R}^{3}\right)\right)^{3},
$$

thus the right hand side of (1.7) is the first variation functional of $V(t)$ at $\varphi$.

We will show that also we have $\nabla \chi \in L_{w^{*}}^{\infty}\left(0, T,\left(\mathcal{M}\left(\mathbb{R}^{3}\right)\right)^{3}\right)$ and the connection between the time dependent 2-varifold $V$ and $\chi$ is given by the equation

$$
-\int_{\mathbb{R}^{3}} \varphi \cdot \nabla \chi(t)(d x) d t=\int_{\mathbb{R}^{3} \times \mathbb{S}^{2}} \varphi \cdot y V(t)(d(x, y)), \quad \forall \varphi \in\left(C_{c}^{\infty}\left(\mathbb{R}^{3}\right)\right)^{3} \quad \text { for a.e. } t \in(0, T) .
$$




\subsection{Axisymmetry}

For an angle $\theta \in \mathbb{R}$ let us denote by $J(\theta)$ the rotation matrix with the angle $\theta$ in $\mathbb{R}^{2}$ and by $O(\theta)$ the rotation matrix with the angle $\theta$ around the axis $e_{3}$ in $\mathbb{R}^{3}$, i.e.

$$
J(\theta)=\left[\begin{array}{rr}
\cos (\theta) & -\sin (\theta) \\
\sin (\theta) & \cos (\theta)
\end{array}\right] \text { and } O(\theta)=\left[\begin{array}{lr}
J(\theta) & 0_{2} \\
0_{2}^{T} & 1
\end{array}\right]
$$

here $0_{2}$ is the 0 in $\mathbb{R}^{2}$.

We call $h: \mathbb{R}^{3} \rightarrow \mathbb{R}$ axisymmetric if

$$
h(x)=h\left(O^{T}(\theta) x\right), \quad \forall x \in \mathbb{R}^{3} \text { and } \theta \in \mathbb{R} .
$$

We call $w: \mathbb{R}^{3} \rightarrow \mathbb{R}^{3}$ axisymmetric if

$$
w(x)=O(\theta) w\left(O^{T}(\theta) x\right), \quad \forall x \in \mathbb{R}^{3} \text { and } \theta \in \mathbb{R} .
$$

We call $\mathcal{V} \in \mathcal{M}\left(\mathbb{R}^{3} \times \mathbb{S}^{2}\right)$ axisymmetric if

$$
\mathcal{V}(A \times B)=\mathcal{V}\left(\left(O^{T}(\theta) A\right) \times\left(O^{T}(\theta) B\right)\right), \quad \forall A \in \mathcal{B}\left(\mathbb{R}^{3}\right), B \in \mathcal{B}\left(\mathbb{S}^{2}\right) \text { and } \theta \in \mathbb{R}
$$

and

$$
\mathcal{V}\left(\left\{(x, y) \in \mathbb{R}^{3} \times \mathbb{S}^{2} \mid y \cdot\left(e_{3} \times x\right) \neq 0\right\}\right)=0 .
$$

\subsection{Main Results}

Let us define

$$
E^{0}=\text { closure of }\left\{v \in\left(C_{c}^{\infty}\left(\mathbb{R}^{3}\right)\right)^{3} \mid \operatorname{div}(v)=0\right\} \quad \text { in }\left(L^{2}\left(\mathbb{R}^{3}\right)\right)^{3}
$$

and

$$
E^{1}=\text { closure of }\left\{v \in\left(C_{c}^{\infty}\left(\mathbb{R}^{3}\right)\right)^{3} \mid \operatorname{div}(v)=0\right\} \quad \text { in }\left(H^{1}\left(\mathbb{R}^{3}\right)\right)^{3} .
$$

In the statement of the theorem below we will encounter the spaces $L_{w^{*}}^{\infty}\left(0, T,\left(\mathcal{M}\left(\mathbb{R}^{3}\right)\right)^{3}\right)$ and $L_{w^{*}}^{\infty}\left(0, T, \mathcal{M}\left(\mathbb{R}^{3} \times \mathbb{S}^{2}\right)\right)$. These are defined in Sect. 2 .

The main results of this paper are the following two theorems.

Theorem 1.1. Let $u_{0} \in E^{0}, \chi_{0}=1_{\Omega_{1,0}} \in B V\left(\mathbb{R}^{3}\right)$, where

$$
B V\left(\mathbb{R}^{3}\right)=\left\{\eta \in L^{1}\left(\mathbb{R}^{3}\right) \mid \nabla \eta \in\left(\mathcal{M}\left(\mathbb{R}^{3}\right)\right)^{3}\right\}
$$

denotes the space of functions with bounded variation in $\mathbb{R}^{3}$.

Then there exists a triple $(u, \chi, V)$ such that

$$
\begin{aligned}
& u \in L^{\infty}\left(0, T, E^{0}\right) \cap L^{2}\left(0, T, E^{1}\right), \\
& \quad \chi \in L^{\infty}\left(0, T, L^{1}\left(\mathbb{R}^{3} ;\{0,1\}\right)\right), \quad \nabla \chi \in L_{w^{*}}^{\infty}\left(0, T,\left(\mathcal{M}\left(\mathbb{R}^{3}\right)\right)^{3}\right)
\end{aligned}
$$

and

$$
V \in L_{w^{*}}^{\infty}\left(0, T, \mathcal{M}\left(\mathbb{R}^{3} \times \mathbb{S}^{2}\right)\right)
$$

with

$$
\begin{aligned}
& \|u\|_{L^{\infty}\left(0, T, E^{0}\right)}^{2}+\|u\|_{L^{2}\left(0, T, E^{1}\right)}^{2}+\|\chi\|_{L^{\infty}\left(0, T, L^{1}\left(\mathbb{R}^{3} ;\{0,1\}\right)\right)} \\
& \quad+\|\nabla \chi\|_{L_{w^{*}}^{\infty}\left(0, T,\left(\mathcal{M}\left(\mathbb{R}^{3}\right)\right)^{3}\right)}+\|V\|_{L_{w^{*}}^{\infty}\left(0, T, \mathcal{M}\left(\mathbb{R}^{3} \times \mathbb{S}^{2}\right)\right)} \\
& \leq C\left(\left\|\chi_{0}\right\|_{B V\left(\mathbb{R}^{3}\right)}+\left\|u_{0}\right\|_{E^{0}}^{2}\right) .
\end{aligned}
$$

$\chi$ is the renormalized solution of

$$
\begin{cases}\partial_{t} \chi+u \cdot \nabla \chi=0 & \text { in }(0, T) \times \mathbb{R}^{3} \\ \chi(0)=\chi_{0} & \text { in } \mathbb{R}^{3}\end{cases}
$$


The connection between $\nabla \chi$ and $V$ is given by the Eq. (1.10).

For all $\varphi \in\left(C_{c}^{\infty}\left((-\infty, T) \times \mathbb{R}^{3}\right)\right)^{3}$ with $\operatorname{div}(\varphi)=0$, u satisfies

$$
\begin{aligned}
& -\int_{\mathbb{R}^{3}} u_{0}^{T} \varphi(0) d x-\int_{0}^{T} \int_{\mathbb{R}^{3}}\left\{u^{T} \partial_{t} \varphi+(u \otimes u): \nabla \varphi\right\} d x d t+\int_{0}^{T} \int_{\mathbb{R}^{3}} D u: D \varphi d x d t \\
& =-\int_{0}^{T}\langle\delta V(t), \varphi(t)\rangle d t .
\end{aligned}
$$

The solution triple $(u, \chi, V)$ is called a varifold solution. Fo the notion of renormalized solution of the transport equation one may refer to [6].

Theorem 1.2. If in the Theorem 1.1 the initial values $u_{0}$ and $\chi_{0}$ are axisymmetric then there exists a varifold solution $(u, \chi, V)$ with each component being axisymmetric, i.e. for a.e. $0<t<T, \chi(t)$ satisfies (1.11), $u(t)$ satisfies (1.12) and $V(t)$ satisfies (1.13) and (1.14).

\subsection{Organization of this Paper}

This paper is organized as follows, in Sect. 2 we have collected some definitions and facts about Banach space valued functions, in Sect. 3 we consider an approximate regularized problem and using the LeraySchauder principle (which is sometimes called Schaefer's fixed point theorem) we prove the existence of a regularized solution. In Sect. 4 we prove the existence of a varifold solution as the limit of regularized solutions.

\section{Some Facts About Banach Space Valued Functions}

For the following definitions and facts a good reference is [5].

Let us denote by $\lambda$ the Lebesgue measure defined on the Borel subsets of $\mathbb{R}$.

Let $X$ be a Banach space and $I \subset \mathbb{R}$ a bounded interval. Let us consider $f: I \rightarrow X$, then one may consider the following three kinds of measurabilities of $f$.

$f$ is called $\lambda$-measurable if there exists a sequence of simple functions $s_{n}: I \rightarrow X$ such that $s_{n}(t) \rightarrow f(t)$ in $X$ for $\lambda$-a.e. $t \in I$.

$f$ is called weakly- $\lambda$-measurable if for any $g \in X^{*}$ the function (as a function of $\left.t\right)\langle g, f(t)\rangle_{X^{*}, X}$ is measurable.

In the case $X=Y^{*}$ for some Banach space $Y, f$ is called weak ${ }^{*}-\lambda$-measurable if for any $y \in Y$, the function (as a function of $t$ ) $\langle f(t), y\rangle_{Y^{*}, Y}$ is measurable.

The function $f$ is called $\lambda$-essentially separably valued if there exists $E \subset I$ such that $\lambda(E)=0$ and $f(I \backslash E)$ is a separable subset of $X$.

The Pettis measurability theorem states that $f$ is $\lambda$-measurable if and only if $f$ is $\lambda$-essentially separably valued and $f$ is weakly- $\lambda$-measurable.

For $1 \leq p \leq \infty$ by $L^{p}(0, T, X)$ we denote the space of $\lambda$-measurable functions $f:(0, T) \rightarrow X$ such that $\|f(t)\|_{X}$ as a function of $t$ is in $L^{p}(0, T)$.

When $X=Y^{*}$ for some Banach space $Y$, for $1 \leq p \leq \infty$ by $L_{w^{*}}^{p}(0, T, X)$ we denote the space of $w^{*}-\lambda$-measurable functions $f:(0, T) \rightarrow X$ such that also $\|f(t)\|_{X}$ as a function of $t$ is measurable and is in $L^{p}(0, T)$.

If $X$ is a reflexive Banach space and $1 \leq p<\infty$ then $\left(L^{p}(0, T, X)\right)^{*}=L^{q}\left(0, T, X^{*}\right)$ where $p^{-1}+q^{-1}=1$. 


\section{Approximate Regularized Problem}

In Sect. 3.1 we define rotation operators. In Sect. 3.2 we define a regularisation operator of solenoidal time dependent vector fields $\Psi_{\epsilon}$. In the Sect. 3.3 we define the approximate regularised problem and in Sect. 3.4 we prove the existence of a solution to the regularised problem.

\subsection{Rotation Operators}

For a function $h: \mathbb{R}^{3} \rightarrow \mathbb{R}$ and $\theta \in \mathbb{R}$ we define the clockwise rotation around the axis $e_{3}$ of the function $h$ by angle $\theta$ as $\tau_{\theta}(h)(x)=h\left(O^{T}(\theta) x\right)$ for $x \in \mathbb{R}^{3}$. It is easy to see that for a smooth $h$ for $x \in \mathbb{R}^{3}$ we have

$$
\nabla\left(\tau_{\theta} h\right)(x)=O(\theta) \nabla h\left(O^{T}(\theta) x\right) .
$$

For a function $w: \mathbb{R}^{3} \rightarrow \mathbb{R}^{3}$ and $\theta \in \mathbb{R}$ we define the clockwise rotation around the axis $e_{3}$ of the function $w$ by the angle $\theta$ as follows $T_{\theta}(w)(x)=O(\theta) w\left(O^{T}(\theta) x\right)$ for $x \in \mathbb{R}^{3}$. It is easy to see that for a smooth $w$ for $x \in \mathbb{R}^{3}$ we have

$$
\nabla\left(T_{\theta} \varphi\right)(x)=O(\theta) \nabla \varphi\left(O^{T}(\theta) x\right) O^{T}(\theta) .
$$

We fix a mollifier $\psi \in C_{c}^{\infty}\left(B_{1}^{3}\right)$ such that $\int_{B_{1}^{3}} \psi(x) d x=1$ and is radial i.e. $\psi(x)=\psi(y)$ for $|x|=|y|$. As usual notation for mollifiers $\psi_{\beta}(x)=\beta^{-3} \psi\left(\beta^{-1} x\right)$ for $\beta>0$.

Proposition 3.1. The operators $\psi_{\beta} *$ and $\tau_{\theta}$ commute. The operators $\psi_{\beta} *$ and $T_{\theta}$ commute.

Proof. By direct computations.

\subsection{The Operator $\Psi_{\epsilon}$}

In the rest of this paper, we always have $0<\epsilon<1, \alpha(\epsilon)=\frac{1}{2} \epsilon^{2}$ and $\beta(\epsilon)=\sqrt{\epsilon}$.

In this subsection we define a regularizing and compact linear operator $\Psi_{\epsilon}$ of solenoidal vector fields which preserves axisymmetry. We will use this operator extensively in our regularized problem.

We define

$$
\Psi_{\epsilon}: L^{2}\left(0, T,\left(L^{2}\left(\mathbb{R}^{3}\right)\right)^{3}\right) \rightarrow L^{2}\left(0, T,\left(L^{2}\left(\mathbb{R}^{3}\right)\right)^{3}\right)
$$

as follows

$$
\Psi_{\epsilon}=P K_{\epsilon}
$$

where we define the compact operator $K_{\epsilon}$ below and $P$ is the Helmholtz projection operator which projects $\left(L^{2}\left(\mathbb{R}^{3}\right)\right)^{3}$ on divergence free vector fields in this space.

We fix a mollifier $\phi \in C_{c}^{\infty}(0,1)$ such that $\int_{(0,1)} \phi(t) d t=1$ and $\phi \geq 0$. As usual $\phi_{\alpha}(t)=\alpha^{-1} \phi\left(\alpha^{-1} t\right)$ for $\alpha>0$. It is very crucial that the support of $\phi$ is on the positive numbers because this makes the value of the convolution in time $\left(\phi_{\alpha} * v\right)(t)$ for some function $v$ to depend only on the vales of $v$ in $(-\infty, 0)$, i.e. historic values.

We define the cutoff function in space $\tilde{\psi}_{\beta}(x)=\left(\psi * 1_{B_{\beta^{-1}-1}^{3}}\right)(x)$.

We define the operator $K_{\epsilon}: L^{2}\left(0, T,\left(L^{2}\left(\mathbb{R}^{3}\right)\right)^{3}\right) \rightarrow\left(C_{c}^{\infty}\left(\mathbb{R}^{4}\right)\right)^{3}$ as follows

$$
\left(K_{\epsilon} w\right)(t, x)=\tilde{\psi}_{\beta(\epsilon)}(x)\left(\left(\phi_{\alpha(\epsilon)} \psi_{\beta(\epsilon)}\right) * \bar{w}\right)(t, x)
$$

where $\bar{w}$ is equal to $w$ for $t \in(0, T)$ and equal to 0 on $(0, T)^{c}$.

Proposition 3.2. The operator $K_{\epsilon}$ commutes with $T_{\theta}$. We have

$$
K_{\epsilon}: L^{2}\left(0, T,\left(L^{2}\left(\mathbb{R}^{3}\right)\right)^{3}\right) \rightarrow\left(C_{c}^{\infty}\left(Q_{\epsilon}\right)\right)^{3}
$$

where $Q_{\epsilon}=(0, T+\alpha(\epsilon)) \times B_{\beta(\epsilon)^{-1}}^{3}$. For $k \in \mathbb{N} \cup\{0\}, K_{\epsilon}$ maps $L^{2}\left(0, T,\left(L^{2}\left(\mathbb{R}^{3}\right)\right)^{3}\right)$ continuously into $\left(H_{0}^{k}\left(Q_{\epsilon}\right)\right)^{3}$. 
Proof. One uses the commuting properties outlined in the Proposition 3.1 and the cutoff and mollification structure of $K_{\epsilon}$.

In terms of the Fourier transform (cf. [16]) the Helmholtz projection operator $P:\left(L^{2}\left(\mathbb{R}^{3}\right)\right)^{3} \rightarrow$ $\left(L^{2}\left(\mathbb{R}^{3}\right)\right)^{3}$ might be written as $P w=\mathcal{F}^{-1}(M \mathcal{F}(w))$ where $M(\zeta)=I-|\zeta|^{-2} \zeta \zeta^{T}$ and $\mathcal{F}$ denotes the Fourier transform for functions defined on $\mathbb{R}^{3}$. Also for a function $u$ defined on $\mathbb{R}^{4}$ we denote by $P(u)$ the function $P(u)(t, x)=P(u(t))(x)$ for $t \in \mathbb{R}$ and $x \in \mathbb{R}^{3}$.

Proposition 3.3. The operator $P$ commutes with $T_{\theta}$.

For all $k \in\{0\} \cup \mathbb{N}$ we have $[P(u)]_{\left(H^{k}\left(\mathbb{R}^{3}\right)\right)^{3}} \leq[u]_{\left(H^{k}\left(\mathbb{R}^{3}\right)\right)^{3}}$ for all $u \in\left(H^{k}\left(\mathbb{R}^{3}\right)\right)^{3}$ and similarly $\|P(u)\|_{\left(H^{k}\left(\mathbb{R}^{4}\right)\right)^{3}} \leq\|u\|_{\left(H^{k}\left(\mathbb{R}^{4}\right)\right)^{3}}$ for all $u \in\left(H^{k}\left(\mathbb{R}^{4}\right)\right)^{3}$.

Let $\omega(x)=\Pi_{i=1}^{3}\left(1+\left|x_{i}\right|\right)^{-\frac{1}{2}}$ for $x \in \mathbb{R}^{3}$ then $P$ is a bounded linear operator mapping $\left(L^{2}\left(\mathbb{R}^{3} ; \omega\right)\right)^{3}$ to itself.

Proof. The commuting property of $P$ with $T_{\theta}$ follows from the fact that Fourier transform commutes with $T_{\theta}$ and $M\left(O^{T}(\theta) \zeta\right)=O^{T}(\theta) M(\zeta) O(\theta)$.

We have

$$
|M(\zeta)|_{2,2} \leq 1 \text { for all } \zeta \in \mathbb{R}^{3}
$$

where $|\cdot|_{2,2}$ is the matrix norm when the Euclidean norm for vectors is considered.

Let $k \in\{0\} \cup \mathbb{N}$ then from (3.4) it follows that

$$
[P(u)]_{\left(H^{k}\left(\mathbb{R}^{3}\right)\right)^{3}}^{2}=\int_{\mathbb{R}^{3}}|\zeta|^{2 k}|M(\zeta) \mathcal{F}(u)|^{2} d \zeta \leq[u]_{\left(H^{k}\left(\mathbb{R}^{3}\right)\right)^{3}}^{2} .
$$

Similarly if we denote by $\tilde{\mathcal{F}}$ the Fourier transform of functions defined on $\mathbb{R}^{4}$ then by the separation of variable property of Fourier transform we have actually $P(u)=\tilde{\mathcal{F}}^{-1}(M \tilde{\mathcal{F}}(u))$ for $u \in\left(C_{c}^{\infty}\left(\mathbb{R}^{4}\right)\right)^{3}$ hence again by (3.4) we obtain $\|P(u)\|_{\left(H^{k}\left(\mathbb{R}^{4}\right)\right)^{3}} \leq\|u\|_{\left(H^{k}\left(\mathbb{R}^{4}\right)\right)^{3}}$.

By the definition of $P$ we have for $j \in\{1,2,3\}$

$$
(P(u))_{j}=u_{j}-R_{j}\left(\sum_{k=1}^{3} R_{k}\left(u_{k}\right)\right)
$$

where $R_{j}$ is the $j$ th Riesz transform.

It is known that (cf. [15]) if $\eta \in A_{2}$ where

$$
A_{2}=\left\{\eta \in L_{l o c}^{1}\left(\mathbb{R}^{3}\right) \mid \eta \geq 0, \sup _{B}\left\{\frac{1}{|B|^{2}} \int_{B} \eta d x \int_{B} \frac{1}{\eta} d x\right\}<\infty\right\}
$$

here the supremum is over all balls $B \subset \mathbb{R}^{3}$, then $R_{j}$ is a bounded linear operator of $L^{2}\left(\mathbb{R}^{3} ; \eta\right)$ to itself. Now one may check that $\omega \in A_{2}$ thus by $(3.5)$ we obtain that $P$ is a bounded linear operator mapping $\left(L^{2}\left(\mathbb{R}^{3} ; \omega\right)\right)^{3}$ to itself.

Lemma 3.4. The operators $\Psi_{\epsilon}$ and $T_{\theta}$ commute.

For each $u \in L^{2}\left(0, T,\left(L^{2}\left(\mathbb{R}^{3}\right)\right)^{3}\right), \operatorname{supp}\left(\Psi_{\epsilon}(u)\right) \subset[0, T+\alpha(\epsilon)] \times \mathbb{R}^{3}$.

For each $k \in\{0\} \cup \mathbb{N}, \Psi_{\epsilon}$ is a compact map of $L^{2}\left(0, T,\left(L^{2}\left(\mathbb{R}^{3}\right)\right)^{3}\right)$ to $\left(C_{b}^{k}\left(\mathbb{R}^{4}\right)\right)^{3}$.

$\Psi_{\epsilon}$ is a bounded linear operator mapping $L^{2}\left(0, T,\left(L^{2}\left(\mathbb{R}^{3} ; \omega\right)\right)^{3}\right)$ to itself and its corresponding norm is uniformly bounded with respect to $\epsilon$.

For $u \in L^{2}\left(0, T,\left(L^{2}\left(\mathbb{R}^{3} ; \omega\right)\right)^{3}\right)$ we have $\Psi_{\epsilon}(u) \rightarrow P(u)$ in $L^{2}\left(0, T,\left(L^{2}\left(\mathbb{R}^{3} ; \omega\right)\right)^{3}\right)$ as $\epsilon \rightarrow+0$.

For $u \in L^{2}\left(0, T,\left(H^{1}\left(\mathbb{R}^{3}\right)\right)^{3}\right)$ we have $\Psi_{\epsilon}^{*}(u) \rightarrow P^{*}(u)$ in $\left(L^{2}\left(0, T,\left(H^{1}\left(\mathbb{R}^{3}\right)\right)^{3}\right)\right)^{*}$ as $\epsilon \rightarrow+0$.

Proof. Because $P$ and $K_{\epsilon}$ commute with $T_{\theta}$ we obtain that $\Psi_{\epsilon}$ commutes with $T_{\theta}$.

Fix $k \in\{0\} \cup \mathbb{N}$. Let $m \in \mathbb{N}$ large enough such that by the Sobolev embedding theorem $\left(H^{m}\left(\mathbb{R}^{4}\right)\right)^{3}$ is continuously embedded in the space $\left(C_{b}^{k}\left(\mathbb{R}^{4}\right)\right)^{3}$. The function $K_{\epsilon}$ maps $L^{2}\left(0, T,\left(L^{2}\left(\mathbb{R}^{3}\right)\right)^{3}\right)$ in $H_{0}^{m+1}\left(Q_{\epsilon}\right)$ continuously. Thus by the compact embedding of $H_{0}^{m+1}\left(Q_{\epsilon}\right)$ in $H_{0}^{m}\left(Q_{\epsilon}\right)$ we have that $K_{\epsilon}$ is a compact operator mapping $L^{2}\left(0, T,\left(L^{2}\left(\mathbb{R}^{3}\right)\right)^{3}\right)$ to $H_{0}^{m}\left(Q_{\epsilon}\right)$. The operator $P$ maps $H^{m}\left(\mathbb{R}^{4}\right)$ continuously in itself. 
Finally by the continuous embedding of this space in $\left(C_{b}^{k}\left(\mathbb{R}^{4}\right)\right)^{3}$ we prove the compactness of the operator $\Psi_{\epsilon}$ mapping $L^{2}\left(0, T,\left(L^{2}\left(\mathbb{R}^{3}\right)\right)^{3}\right)$ to $\left(C_{b}^{k}\left(\mathbb{R}^{4}\right)\right)^{3}$.

We have

$$
\Psi_{\epsilon}(u)=\phi_{\alpha(\epsilon)} *\left(P\left(\tilde{\psi}_{\beta(\epsilon)}\left(\psi_{\beta(\epsilon)} * \bar{u}\right)\right)\right) .
$$

Because $\omega \in A_{2}$ we have that $\psi_{\beta} *$ maps $L^{2}\left(\mathbb{R}^{3} ; \omega\right)$ to itself with a norm uniformly bounded with respect to $0<\beta<1$ (cf. [15]). The operator $P$ is also bounded mapping $\left(L^{2}\left(\mathbb{R}^{3} ; \omega\right)\right)^{3}$ to itself as stated in Proposition 3.3. The rest of the operators in $(3.6)$ have also uniformly bounded in $\epsilon$ norms as operators mapping $\left(L^{2}\left(\mathbb{R}^{3} ; \omega\right)\right)^{3}$ to itself or $L^{2}\left(0, T,\left(L^{2}\left(\mathbb{R}^{3} ; \omega\right)\right)^{3}\right)$ to itself. And this proves the claimed boundedness and uniform bound.

The last two claims of the lemma are easy to check.

\subsection{Approximate Regularized Problem}

Let us define $\chi_{0, \epsilon}=\psi_{\epsilon} * \chi_{0}$.

Proposition 3.5. We have

$$
\begin{gathered}
0 \leq \chi_{0, \epsilon} \leq 1 \\
\left\|\chi_{0, \epsilon}\right\|_{L^{1}\left(\mathbb{R}^{3}\right)} \leq\left\|\chi_{0}\right\|_{L^{1}\left(\mathbb{R}^{3}\right)},\left\|\chi_{0, \epsilon}\right\|_{L^{2}\left(\mathbb{R}^{3}\right)} \leq\left\|\chi_{0}\right\|_{L^{2}\left(\mathbb{R}^{3}\right)}, \\
\left\|\nabla \chi_{0, \epsilon}\right\|_{L^{1}\left(\mathbb{R}^{3}\right)} \leq C\left|\nabla \chi_{0}\right|\left(\mathbb{R}^{3}\right)
\end{gathered}
$$

and if $\Omega_{1,0}$ is axisymmetric then $\chi_{0, \epsilon}$ is axisymmetric.

Proof. Because $\chi_{0}(x) \in\{0,1\}$ for all $x \in \mathbb{R}^{3}, \psi \geq 0$ and $\int_{B_{1}^{3}} \psi(x) d x=1$ we obtain (3.7). Using the Young inequality for convolution one obtains the inequalities in (3.8) and (3.9).

If $\Omega_{1,0}$ is axisymmetric then $\chi_{0}$ is axisymmetric. Now because $\psi_{\epsilon} *$ commutes with $\tau_{\theta}, \chi_{0, \epsilon}$ is axisymmetric.

For $\chi \in H^{1}\left(\mathbb{R}^{3}\right)$ we define $\tilde{f}_{\epsilon}^{\text {s.t. }}(\chi) \in\left(\left(H^{1}\left(\mathbb{R}^{3}\right)\right)^{3}\right)^{*}$ by

$$
\left\langle\tilde{f}_{\epsilon}^{\text {s.t. }}(\chi), \varphi\right\rangle=\int_{\mathbb{R}^{3}} \frac{\nabla \chi \otimes \nabla \chi}{\left(|\nabla \chi|^{2}+\epsilon^{2}\right)^{\frac{1}{2}}}: \nabla \varphi d x \quad \text { for } \varphi \in\left(H^{1}\left(\mathbb{R}^{3}\right)\right)^{3}
$$

and for $\chi \in L^{2}\left(0, T, H^{1}\left(\mathbb{R}^{3}\right)\right)$ we define $f_{\epsilon}^{\text {s.t. }}(\chi) \in\left(L^{2}\left(0, T,\left(L^{2}\left(\mathbb{R}^{3}\right)\right)^{3}\right)\right)^{*}$ for $\varphi \in L^{2}\left(0, T,\left(L^{2}\left(\mathbb{R}^{3}\right)\right)^{3}\right)$ by

$$
\left\langle f_{\epsilon}^{\text {s.t. }}(\chi), \varphi\right\rangle=\int_{0}^{T}\left\langle\tilde{f}_{\epsilon}^{\text {s.t. }}(\chi), \Psi_{\epsilon}(\varphi)\right\rangle d t .
$$

Let us denote by $J$ the isomorphism between the spaces $\left(L^{2}\left(0, T,\left(L^{2}\left(\mathbb{R}^{3}\right)\right)^{3}\right)\right)^{*}$ and $L^{2}(0, T$, $\left.\left(\left(L^{2}\left(\mathbb{R}^{3}\right)\right)^{3}\right)^{*}\right)$.

Now we are in the position to state our regularized system of a transport equation

$$
\left\{\begin{array}{l}
\partial_{t} \chi_{\epsilon}+\Psi_{\epsilon}\left(u_{\epsilon}\right) \cdot \nabla \chi_{\epsilon}-\epsilon \Delta \chi_{\epsilon}=0 \quad \text { in }(0, T) \times \mathbb{R}^{3}, \\
\chi_{\epsilon}(0)=\chi_{0, \epsilon}
\end{array}\right.
$$

together with the momentum equation

$$
\left\{\begin{array}{l}
\partial_{t} u_{\epsilon}+\operatorname{div}\left(\Psi_{\epsilon}\left(u_{\epsilon}\right) \otimes u_{\epsilon}\right)-\operatorname{div}\left(D\left(u_{\epsilon}\right)\right)=J\left(f_{\epsilon}^{\text {s.t. }}\left(\chi_{\epsilon}\right)\right) \text { in }(0, T) \times \mathbb{R}^{3}, \\
u_{\epsilon}(0)=u_{0} .
\end{array}\right.
$$

The precise sense in which the equations above should hold will be clear in the following. 


\subsection{Existence of Solution to the Regularized Problem}

In the following lemmas we prove boundedness and continuity properties of the transport equation, the force term and the momentum equation with a prescribed transport term. These results will be used in applying the Leray-Schauder principle.

Let us denote

$$
W=\left\{w \in\left(C_{b}^{\infty}\left(\mathbb{R}^{4}\right)\right)^{3} \mid \operatorname{div}(w)=0\right\}
$$

where $C_{b}^{\infty}\left(\mathbb{R}^{4}\right)=\cap_{k \in\{0\} \cup \mathbb{N}} C_{b}^{k}\left(\mathbb{R}^{4}\right)$.

Lemma 3.6. Let $w \in W$ then the following equation

$$
\begin{cases}\partial_{t} \chi+w \cdot \nabla \chi-\epsilon \triangle \chi=0 & \text { in }(0, T) \times \mathbb{R}^{3} \\ \chi(0)=\chi_{0, \epsilon} & \text { in } \mathbb{R}^{3}\end{cases}
$$

has a unique classical solution $\chi \in C_{b}^{2}\left([0, T) \times \mathbb{R}^{3}\right)$. Let us define the nonlinear operator $G_{\epsilon}(w)=\chi$. We have

$$
0 \leq \chi \leq 1
$$

and

$$
\|\chi\|_{L^{\infty}\left(0, T, L^{2}\left(\mathbb{R}^{3}\right)\right)}+\sqrt{\epsilon}\|\chi\|_{L^{2}\left(0, T, H^{1}\left(\mathbb{R}^{3}\right)\right)} \leq C\left\|\chi_{0, \epsilon}\right\|_{L^{2}\left(\mathbb{R}^{3}\right)} .
$$

Considering the supremum norm for the space $W$, the map

$$
G_{\epsilon}: W \rightarrow L^{2}\left(0, T, H^{1}\left(\mathbb{R}^{3}\right)\right)
$$

is (Lipschitz) continuous.

If $w$ and $\Omega_{1,0}$ are axisymmetric then so is $\chi$.

Proof. The inequalities (3.13) follow from maximum principle and (3.7).

By multiplying the Eq. (3.12) with $\chi$, integrations by part and using that $w \in\left(C_{b}\left(\mathbb{R}^{3}\right)\right)^{3}$ with $\operatorname{div}(w)=0$ we obtain $(3.14)$.

Let $w_{i} \in X$ for $i=1,2$ and $\chi_{i}$ the corresponding solutions to (3.12). Taking the difference of the equations satisfied by $\chi_{i}$ and denoting $w=w_{2}-w_{1}$ and $\chi=\chi_{2}-\chi_{1}$ we obtain

$$
\partial_{t} \chi+\frac{1}{2}\left(w_{1}+w_{2}\right) \cdot \nabla \chi-\epsilon \Delta \chi=-\frac{1}{2} w \cdot \nabla\left(\chi_{2}+\chi_{1}\right)
$$

now this is an equation satisfied by $\chi$ with the initial value $\chi(0)=0$. By similar computations as one does to obtain (3.14) and using the inequality (3.14) for $\chi_{1}$ and $\chi_{2}$ one obtains the inequality $\|\chi\|_{L^{2}\left(0, T, H^{1}\left(\mathbb{R}^{3}\right)\right)} \leq C_{\epsilon}\left\|\chi_{0, \epsilon}\right\|_{L^{2}\left(\mathbb{R}^{3}\right)}\|w\|_{C_{b}\left(\mathbb{R}^{4}\right)}$ which proves the Lipschitz continuity of $G_{\epsilon}$.

If $\Omega_{1,0}$ is axisymmetric then by Proposition $3.5, \chi_{0, \epsilon}$ is axisymmetric. If also $w$ is axisymmetric it is easy to see that for any $\theta \in \mathbb{R}$, the function $\tau_{\theta}(\chi)$ is also a solution to our equation and hence by uniqueness we should have $\chi=\tau_{\theta}(\chi)$ for all $\theta \in \mathbb{R}$ which proves that $\chi$ is axisymmetic.

We call $f \in\left(L^{2}\left(0, T,\left(L^{2}\left(\mathbb{R}^{3}\right)\right)^{3}\right)\right)^{*}$ axisymmetric if $T_{\theta}^{*} f=f$ for all $\theta \in \mathbb{R}$, i.e. $\langle f, \varphi\rangle=\left\langle f, T_{\theta} \varphi\right\rangle$ for all $\varphi \in L^{2}\left(0, T,\left(L^{2}\left(\mathbb{R}^{3}\right)\right)^{3}\right)$ and $\theta \in \mathbb{R}$.

Lemma 3.7. The function $f_{\epsilon}^{\text {s.t. }:} L^{2}\left(0, T, H^{1}\left(\mathbb{R}^{3}\right)\right) \rightarrow\left(L^{2}\left(0, T,\left(L^{2}\left(\mathbb{R}^{3}\right)\right)^{3}\right)\right)^{*}$ is continuous and if $\chi$ is axisymmetric then $f_{\epsilon}^{\text {s.t. }}(\chi)$ is axisymmetric.

Proof. Let $\varphi \in L^{2}\left(0, T,\left(L^{2}\left(\mathbb{R}^{3}\right)\right)^{3}\right)$. Let $\chi_{i} \in L^{2}\left(0, T, H^{1}\left(\mathbb{R}^{3}\right)\right)$ for $i=1,2$ then

$$
\begin{aligned}
& \left\langle f_{\epsilon}^{\text {s.t. }}\left(\chi_{2}\right)-f_{\epsilon}^{\text {s.t. }}\left(\chi_{1}\right), \varphi\right\rangle \\
& \quad=\int_{0}^{T} \int_{\mathbb{R}^{3}}\left(\frac{\partial_{i} \chi_{2} \partial_{j} \chi_{2}}{\left(\left|\nabla \chi_{2}\right|^{2}+\epsilon^{2}\right)^{\frac{1}{2}}}-\frac{\partial_{i} \chi_{1} \partial_{j} \chi_{1}}{\left(\left|\nabla \chi_{1}\right|^{2}+\epsilon^{2}\right)^{\frac{1}{2}}}\right) \partial_{x_{i}}\left(\Psi_{\epsilon}(\varphi)\right)_{j} d x d t .
\end{aligned}
$$

Let us denote for $p, q \in \mathbb{R}$ and $a>0, A(p, q, a)=\left(a^{2}+\epsilon^{2}\right)^{-\frac{1}{2}} p q$ then we have $\partial_{p} A(p, q, a)=$ $\left(a^{2}+\epsilon^{2}\right)^{-\frac{1}{2}} q, \partial_{q} A(p, q, a)=\left(a^{2}+\epsilon^{2}\right)^{-\frac{1}{2}} p$ and $\partial_{a} A(p, q, a)=-\left(a^{2}+\epsilon^{2}\right)^{-\frac{3}{2}} p q a$. So for $|p|,|q| \leq a$ we have 
$\left|\partial_{p} A(p, q, a)\right| \leq 1,\left|\partial_{q} A(p, q, a)\right| \leq 1$ and $\left|\partial_{a} A(p, q, a)\right| \leq 1$. Now let $\left|p_{k}\right|,\left|q_{k}\right| \leq a_{k}$ for $k=1,2$ then for $0 \leq \tau \leq 1$ we have $\left|(1-\tau) p_{1}+\tau p_{2}\right| \leq(1-\tau)\left|p_{1}\right|+\tau\left|p_{2}\right| \leq(1-\tau) a_{1}+\tau a_{2}$ and similarly for $q_{k}$ hence we can estimate using the mean value theorem $\left|A\left(p_{2}, q_{2}, a_{2}\right)-A\left(p_{1}, q_{1}, a_{1}\right)\right| \leq\left|p_{2}-p_{1}\right|+\left|q_{2}-q_{1}\right|+\left|a_{2}-a_{1}\right|$.

Considering $p_{k}=\partial_{x_{i}} \chi_{k}, q_{k}=\partial_{x_{j}} \chi_{k}$ and $a_{k}=\left|\nabla \chi_{k}\right|$ we have

$$
\begin{aligned}
& \left|A\left(\partial_{x_{i}} \chi_{2}, \partial_{x_{j}} \chi_{2},\left|\nabla \chi_{2}\right|\right)-A\left(\partial_{x_{i}} \chi_{1}, \partial_{x_{j}} \chi_{1},\left|\nabla \chi_{1}\right|\right)\right| \\
& \quad \leq\left\{\left|\partial_{x_{i}} \chi_{2}-\partial_{x_{i}} \chi_{1}\right|+\left|\partial_{x_{j}} \chi_{2}-\partial_{x_{j}} \chi_{1}\right|+|| \nabla \chi_{2}|-| \nabla \chi_{1}||\right\} \leq 3\left|\nabla \chi_{2}-\nabla \chi_{1}\right| .
\end{aligned}
$$

Now by (3.15), the definition of $A$, Eq. (3.16) and Lemma 3.4 we obtain

$$
\begin{aligned}
\left|\left\langle f_{\epsilon}^{\text {s.t. }}\left(\chi_{2}\right)-f_{\epsilon}^{\text {s.t. }}\left(\chi_{1}\right), \varphi\right\rangle\right| & \leq C \int_{0}^{T} \int_{\mathbb{R}^{3}}\left|\nabla \chi_{2}-\nabla \chi_{1}\right|\left|\nabla \Psi_{\epsilon}(\varphi)\right| d x d t \\
& \leq C\left\{\int_{0}^{T} \int_{\mathbb{R}^{3}}\left|\nabla \chi_{2}-\nabla \chi_{1}\right|^{2} d x d t\right\}^{\frac{1}{2}}\left\{\int_{0}^{T} \int_{\mathbb{R}^{3}}\left|\nabla \Psi_{\epsilon}(\varphi)\right|^{2} d x d t\right\}^{\frac{1}{2}} .
\end{aligned}
$$

Denoting $v=\nabla P \tilde{\psi}_{\beta(\epsilon)} \psi_{\beta(\epsilon)} * \bar{\varphi}$ and using two times the Minkowski inequality, separately we estimate

$$
\begin{aligned}
& \int_{0}^{T} \int_{\mathbb{R}^{3}}\left|\nabla \Psi_{\epsilon}(\varphi)\right|^{2} d x d t=\int_{0}^{T} \int_{\mathbb{R}^{3}}\left|\phi_{\alpha(\epsilon)} * v\right|^{2} d x d t \\
& \leq \int_{0}^{T}\left\|\phi_{\alpha(\epsilon)} * v\right\|_{\left(L^{2}\left(\mathbb{R}^{3}\right)\right)^{3 \times 3}}^{2} d t \leq \int_{0}^{T}\left(\phi_{\alpha(\epsilon)} *\|v\|_{\left.\left(L^{2}\left(\mathbb{R}^{3}\right)\right)^{3 \times 3}\right)^{2}} d t\right. \\
& =\int_{0}^{T}\left(\int_{0}^{T} \phi_{\alpha(\epsilon)}(s)\|v\|_{\left(L^{2}\left(\mathbb{R}^{3}\right)\right)^{3 \times 3}}(t-s) d s\right)^{2} d t \\
& \leq\left\{\int_{0}^{T} \phi_{\alpha(\epsilon)}(s)\left\{\int_{0}^{T}\|v\|_{\left(L^{2}\left(\mathbb{R}^{3}\right)\right)^{3 \times 3}}^{2}(t-s) d t\right\}^{\frac{1}{2}} d s\right\}^{2} \\
& =\left\{\int_{0}^{\alpha(\epsilon)} \phi_{\alpha(\epsilon)}(s)\left\{\int_{-s}^{T-s}\|v\|_{\left(L^{2}\left(\mathbb{R}^{3}\right)\right)^{3 \times 3}}^{2}(z) d z\right\}^{\frac{1}{2}} d s\right\}^{2} \\
& \leq\left\{\int_{0}^{\alpha(\epsilon)} \phi_{\alpha(\epsilon)}(s) d s\right\}^{2} \int_{0}^{T}\|v\|_{\left(L^{2}\left(\mathbb{R}^{3}\right)\right)^{3 \times 3}}^{2}(z) d z \\
& \leq \int_{0}^{T}\|v\|_{\left(L^{2}\left(\mathbb{R}^{3}\right)\right)^{3 \times 3}}^{2}(z) d z \\
& =\int_{0}^{T}\left[P \tilde{\psi}_{\beta(\epsilon)} \psi_{\beta(\epsilon)} * \bar{\varphi}\right]_{\left(H^{1}\left(\mathbb{R}^{3}\right)\right)^{3}}^{2}(z) d z \\
& \leq C \int_{0}^{T}\left[\tilde{\psi}_{\beta(\epsilon)} \psi_{\beta(\epsilon)} * \bar{\varphi}\right]_{\left(H^{1}\left(\mathbb{R}^{3}\right)\right)^{3}}^{2}(z) d z \\
& \leq C_{\epsilon} \int_{0}^{T}\|\varphi(t)\|_{\left(L^{2}\left(\mathbb{R}^{3}\right)\right)^{3}}^{2} d t \leq C_{\epsilon}\|\varphi\|_{L^{2}\left(0, T,\left(L^{2}\left(\mathbb{R}^{3}\right)\right)^{3}\right)}^{2}
\end{aligned}
$$

and this together with (3.17) proves the continuity of $f_{\epsilon}^{\text {s.t. }}$.

Let $\chi \in L^{2}\left(0, T, H^{1}\left(\mathbb{R}^{3}\right)\right)$ be axisymmetric, $\varphi \in L^{2}\left(0, T,\left(L^{2}\left(\mathbb{R}^{3}\right)\right)^{3}\right)$ and $\theta \in \mathbb{R}$ then by Lemma 3.4, Eqs. (3.1) and (3.2)

$$
\left\langle f_{\epsilon}^{\text {s.t. }}(\chi), T_{\theta} \varphi\right\rangle=\int_{0}^{T} \int_{\mathbb{R}^{3}} \frac{\nabla \chi^{T} \nabla\left(\Psi_{\epsilon}\left(T_{\theta} \varphi\right)\right) \nabla \chi}{\left(|\nabla \chi|^{2}+\epsilon^{2}\right)^{\frac{1}{2}}} d x d t
$$




$$
\begin{aligned}
& =\int_{0}^{T} \int_{\mathbb{R}^{3}} \frac{\nabla \chi^{T} \nabla\left(T_{\theta}\left(\Psi_{\epsilon}(\varphi)\right)\right) \nabla \chi}{\left(|\nabla \chi|^{2}+\epsilon^{2}\right)^{\frac{1}{2}}} d x d t \\
& =\int_{0}^{T} \int_{\mathbb{R}^{3}} \frac{\nabla \chi^{T}\left(O^{T}(\theta) \nabla\left(\Psi_{\epsilon}(\varphi)\right)(t, O(\theta) x) O(\theta)\right) \nabla \chi}{\left(|\nabla \chi|^{2}+\epsilon^{2}\right)^{\frac{1}{2}}} d x d t \\
& =\int_{0}^{T} \int_{\mathbb{R}^{3}} \frac{\left(O(\theta) \nabla \chi\left(t, O^{T}(\theta) x\right)\right)^{T} \nabla\left(\Psi_{\epsilon}(\varphi)\right)(t, x) O(\theta) \nabla \chi\left(t, O^{T}(\theta) x\right)}{\left(\left|\nabla \chi\left(t, O^{T}(\theta) x\right)\right|^{2}+\epsilon^{2}\right)^{\frac{1}{2}}} d x d t \\
& =\int_{0}^{T} \int_{\mathbb{R}^{3}} \frac{\left(\nabla \tau_{\theta} \chi\right)^{T} \nabla\left(\Psi_{\epsilon}(\varphi)\right)\left(\nabla \tau_{\theta} \chi\right)}{\left(\mid \nabla\left(\left.\tau_{\theta} \chi\right|^{2}+\epsilon^{2}\right)^{\frac{1}{2}}\right.} d x d t \\
& =\int_{0}^{T} \int_{\mathbb{R}^{3}} \frac{\nabla \chi^{T} \nabla\left(\Psi_{\epsilon}(\varphi)\right) \nabla \chi}{\left(|\nabla \chi|^{2}+\epsilon^{2}\right)^{\frac{1}{2}}} d x d t=\left\langle f_{\epsilon}^{s . t .}(\chi), \varphi\right\rangle
\end{aligned}
$$

which proves that $f_{\epsilon}^{s . t .}$ is axisymmetric.

Lemma 3.8. Let $w \in W$ and $f \in L^{2}\left(0, T,\left(E^{1}\right)^{*}\right)$ then there exists a unique $u \in C\left([0, T), E^{0}\right) \cap L^{2}\left(0, T, E^{1}\right)$ such that $\partial_{t} u \in L^{2}\left(0, T,\left(E^{1}\right)^{*}\right)$ and

$$
\left\{\begin{array}{l}
\partial_{t} u+\operatorname{div}(w \otimes u)-\operatorname{div}(D u)=f \quad \text { in }\left(E^{1}\right)^{*} \quad \text { for a.e. } t \in(0, T), \\
u(0)=u_{0} .
\end{array}\right.
$$

Let us denote $u=A(w, f)$ then $A: W \times L^{2}\left(0, T,\left(E^{1}\right)^{*}\right) \rightarrow L^{2}\left(0, T, E^{1}\right)$ is continuous considering the $\left(C_{b}\left(\mathbb{R}^{4}\right)\right)^{3}$ norm for $W$.

If $w$ and $f$ are axisymmetric then $u=A(w, f)$ is axisymmetric.

Proof. We divide the proof in four steps. In the first step using the Galerkin method we prove the existence of the solution, in the second step we prove the uniqueness of the solution, in the third step we prove the continuous dependence of the solution on the data and finally in the fourth step we show that if $w$ and $f$ are axisymmetric then so is $u$.

Step 1. Existence.

Let the set of the functions $v_{k} \in\left(C_{c}^{\infty}\left(\mathbb{R}^{3}\right)\right)^{3}$ for $k \in \mathbb{N}$ with $\operatorname{div}\left(v_{k}\right)=0$ form a linearly independent and complete subset of $E^{0}$. As usual for the Galerkin method, for each $n \in \mathbb{N}$ we first seek a solution $u_{n}(t, x)=\sum_{k=1}^{n} d_{k}^{n}(t) v_{k}(x)$ which satisfies the equation if the equation is tested only with the functions $v_{k}$ for $k=1, \ldots, n$ and

$$
\left(u_{n}(0), v_{\ell}\right)_{E^{0}}=\left(u_{0}, v_{\ell}\right)_{E^{0}} \quad \text { for } \ell=1, \ldots, n .
$$

Using the fact that $w \in\left(C_{b}\left(\mathbb{R}^{4}\right)\right)^{3}, \chi \in C_{b}^{2}\left([0, T) \times \mathbb{R}^{3}\right)$ and $f \in L^{2}\left(0, T,\left(E^{1}\right)^{*}\right)$ we obtain a well defined ordinary differential equation for $d_{n}$ and obtain a unique solution $d^{n} \in H^{1}\left(0, T, \mathbb{R}^{n}\right)$.

Now our aim is to obtain uniform in $n$ estimates and then to obtain a convergent subsequence of $u_{n}$. Testing the equation satisfied by $u_{n}$ by itself we obtain

$$
\frac{d}{d t} \int_{\mathbb{R}^{3}}\left|u_{n}\right|^{2} d x+\int_{\mathbb{R}^{3}}\left|D u_{n}\right|^{2} d x=\left\langle f(t), u_{n}\right\rangle
$$

By integration in time and using the fact that $f \in L^{2}\left(0, T,\left(E^{1}\right)^{*}\right)$ we obtain $\sup _{n \geq 1}\left\{\left\|u_{n}\right\|_{L^{\infty}\left(0, T, E^{0}\right)}+\right.$ $\left.\left\|u_{n}\right\|_{L^{2}\left(0, T, E^{1}\right)}\right\}<\infty$. So there exists a subsequence $n_{m}$ and

$$
u \in L^{\infty}\left(0, T, E^{0}\right) \cap L^{2}\left(0, T, E^{1}\right)
$$

such that

$$
u_{n_{m}} \stackrel{w^{*}}{\longrightarrow} u \text { in } L^{\infty}\left(0, T, E^{0}\right) \text { and } \quad u_{n_{m}} \stackrel{w}{\longrightarrow} u \quad \text { in } L^{2}\left(0, T, E^{1}\right) .
$$


Fix $\varphi \in\left(C_{c}^{\infty}\left(\mathbb{R}^{4}\right)\right)^{3}$ with $\operatorname{div}(\varphi)=0$. By appropriate approximation of $\varphi$ and passing to the limit in the equation satisfied by $u_{n_{m}}$ we obtain

$$
\begin{aligned}
& -\int_{\mathbb{R}^{3}} u_{0}^{T} \varphi(0) d x-\int_{0}^{T} \int_{\mathbb{R}^{3}} u^{T} \partial_{t} \varphi d x d t-\int_{0}^{T} \int_{\mathbb{R}^{3}} w_{i}(u)_{j} \partial_{x_{i}} \varphi_{j} d x d t+\int_{0}^{T} \int_{\mathbb{R}^{3}} D u: D \varphi d x d t \\
& =\int_{0}^{T}\langle f, \varphi\rangle d t
\end{aligned}
$$

In particular considering (3.21) for $\varphi \in\left(C_{c}^{\infty}\left((0, T) \times \mathbb{R}^{3}\right)\right)^{3}$ with $\operatorname{div}(\varphi)=0$ we obtain that

$$
\partial_{t} u \in L^{2}\left(0, T,\left(E^{1}\right)^{*}\right) .
$$

From (3.20) and (3.22) it follows that $u \in C_{b}\left([0, T], E^{0}\right)$ (cf. [7]). Finally from this continuity and the Eq. (3.21) we obtain $u(0)=u_{0}$.

Step 2. Uniqueness.

If $u_{1}$ and $u_{2}$ are solutions then denoting $u=u_{2}-u_{1}$ we get

$$
\partial_{t} u+\operatorname{div}(w \otimes u)-\operatorname{div}(D u)=0 \quad \text { in }\left(E^{1}\right)^{*} \quad \text { for a.e. } t \in(0, T)
$$

and $u(0)=0$. Then by similar estimates as done above to show the uniform boundedness of $u_{n}$ we obtain that $u=0$.

Step 3. Continuous dependence on the data.

Let $u_{1}$ and $u_{2}$ be solutions corresponding to the pairs $\left(w_{1}, f_{1}\right)$ and $\left(w_{2}, f_{2}\right)$, then denoting $u=u_{2}-u_{1}$ and taking the difference of equations satisfied by $u_{1}$ and $u_{2}$ we obtain

$$
\partial_{t} u+\operatorname{div}\left(\left(\frac{w_{2}+w_{1}}{2}\right) \otimes u\right)-\operatorname{div}(D u)=\left(f_{2}-f_{1}\right)-\operatorname{div}\left(\left(w_{2}-w_{1}\right) \otimes\left(\frac{u_{1}+u_{2}}{2}\right)\right)
$$

in $\left(E^{1}\right)^{*}$ for a.e. $0<t<T$ and $u(0)=0$. By multiplying the equation above by $u$ and proceeding as above to get the uniform bounds on $u_{n}$ we obtain

$$
\begin{aligned}
& \frac{d}{d t} \int_{\mathbb{R}^{3}}|u|^{2} d x+\int_{\mathbb{R}^{3}}|D u|^{2} d x \\
& \quad=\left\langle\left(f_{2}-f_{1}\right)(t), u\right\rangle+\int_{\mathbb{R}^{3}}\left(w_{2}-w_{1}\right)_{i} \partial_{x_{i}} u_{j}\left(\frac{u_{1}+u_{2}}{2}\right)_{j} d x .
\end{aligned}
$$

Now by estimating the right hand side from above and integration in time we obtain

$$
\begin{aligned}
\|u\|_{L^{\infty}\left(0, T, E^{0}\right)}+\|u\|_{L^{2}\left(0, T, E^{1}\right)} \leq & C\left(\left\|f_{2}-f_{1}\right\|_{L^{2}\left(0, T,\left(E^{1}\right)^{*}\right)}\right. \\
& \left.+\left(\left\|u_{1}\right\|_{L^{2}\left(0, T, E^{0}\right)}+\left\|u_{2}\right\|_{L^{2}\left(0, T, E^{0}\right)}\right)\left\|w_{2}-w_{1}\right\|_{\left(C_{b}\left(\mathbb{R}^{4}\right)\right)^{3}}\right) .
\end{aligned}
$$

And by the uniform bounds on $u_{2}$ and $u_{1}$ in $L^{2}\left(0, T, E^{0}\right)$ we obtain the continuity of $A$.

Step 4. If $w$ and $f$ are axisymmetric then so is $u$.

Let $w$ and $f$ be axisymmetric and $\theta \in \mathbb{R}$. One may see that $T_{\theta} u$ is also a solution and thus by uniqueness of the solution we have $u=T_{\theta} u$. Because this holds for all $\theta \in \mathbb{R}, u$ is axisymmetric.

Lemma 3.9. Let $w \in W$ and $\chi=G_{\epsilon}(w)$ then we have

$$
\int_{\mathbb{R}^{3}} \frac{\nabla \chi \otimes \nabla \chi}{\left(|\nabla \chi|^{2}+\epsilon^{2}\right)^{\frac{1}{2}}}: \nabla w d x \leq-\frac{d}{d t} \int_{\mathbb{R}^{3}}\left(\left(|\nabla \chi|^{2}+\epsilon^{2}\right)^{\frac{1}{2}}-\epsilon\right) d x
$$


Proof. Differentiating the equation satisfied by $\chi$ with respect the $x_{i}$, multiplying by $\left(|\nabla \chi|^{2}+\epsilon^{2}\right)^{-\frac{1}{2}} \partial_{x_{i}} \chi$, summing over $i$ and integrating we obtain

$$
\begin{gathered}
\int_{\mathbb{R}^{3}}\left\{\partial_{t} \partial_{x_{i}} \chi \partial_{x_{i}} \chi+\partial_{x_{i}} w_{j} \partial_{x_{j}} \chi \partial_{x_{i}} \chi+w_{j} \partial_{x_{j} x_{i}} \chi \partial_{x_{i}} \chi\right. \\
\left.-\epsilon \triangle \partial_{x_{i}} \chi \partial_{x_{i}} \chi\right\}\left(|\nabla \chi|^{2}+\epsilon^{2}\right)^{-\frac{1}{2}} d x=0
\end{gathered}
$$

now separately we evaluate each term in this equation.

We have

$$
\begin{gathered}
\frac{\partial_{t} \partial_{x_{i}} \chi \partial_{x_{i}} \chi}{\left(|\nabla \chi|^{2}+\epsilon^{2}\right)^{\frac{1}{2}}}=\frac{d}{d t}\left(\left(|\nabla \chi|^{2}+\epsilon^{2}\right)^{\frac{1}{2}}-\epsilon\right), \\
\int_{\mathbb{R}^{3}} \frac{\partial_{x_{i}} w_{j} \partial_{x_{j}} \chi \partial_{x_{i}} \chi}{\left(|\nabla \chi|^{2}+\epsilon^{2}\right)^{\frac{1}{2}}} d x=\int_{\mathbb{R}^{3}} \frac{\nabla \chi \otimes \nabla \chi}{\left(|\nabla \chi|^{2}+\epsilon^{2}\right)^{\frac{1}{2}}}: \nabla w d x
\end{gathered}
$$

and

$$
\int_{\mathbb{R}^{3}} \frac{w_{j} \partial_{x_{j} x_{i}} \chi \partial_{x_{i}} \chi}{\left(|\nabla \chi|^{2}+\epsilon^{2}\right)^{\frac{1}{2}}} d x=\int_{\mathbb{R}^{3}} w_{j} \partial_{x_{j}}\left(\left(|\nabla \chi|^{2}+\epsilon^{2}\right)^{\frac{1}{2}}\right) d x=0
$$

so it remains to evaluate the last term involving the laplacian.

We compute

$$
\begin{aligned}
- & \int_{\mathbb{R}^{3}} \epsilon \Delta \partial_{x_{i}} \chi \frac{\partial_{x_{i}} \chi}{\left(|\nabla \chi|^{2}+\epsilon^{2}\right)^{\frac{1}{2}}} d x=\epsilon \int_{\mathbb{R}^{3}} \partial_{x_{i} x_{j}} \chi \partial_{x_{j}}\left(\frac{\partial_{x_{i}} \chi}{\left(|\nabla \chi|^{2}+\epsilon^{2}\right)^{\frac{1}{2}}}\right) d x \\
= & \epsilon \int_{\mathbb{R}^{3}} \partial_{x_{i} x_{j}} \chi\left\{\frac{\partial_{x_{i} x_{j}} \chi}{\left(|\nabla \chi|^{2}+\epsilon^{2}\right)^{\frac{1}{2}}}-\frac{\partial_{x_{i}} \chi \partial_{x_{k}} \chi \partial_{x_{k} x_{j}} \chi}{\left(|\nabla \chi|^{2}+\epsilon^{2}\right)^{\frac{3}{2}}}\right\} d x \\
= & \epsilon \int_{\mathbb{R}^{3}}\left\{\frac{\operatorname{tr}\left(\nabla^{2} \chi^{T} \nabla^{2} \chi\right)}{\left(|\nabla \chi|^{2}+\epsilon^{2}\right)^{\frac{1}{2}}}-\frac{\nabla^{T} \chi \nabla^{2} \chi^{T} \nabla^{2} \chi \nabla \chi}{\left(|\nabla \chi|^{2}+\epsilon^{2}\right)^{\frac{3}{2}}}\right\} d x \\
= & \epsilon \int_{\mathbb{R}^{3} \cap\{\nabla \chi \neq 0\}} \frac{|\nabla \chi|^{2}}{\left(|\nabla \chi|^{2}+\epsilon^{2}\right)^{\frac{3}{2}}}\left\{\frac{\epsilon^{2}}{|\nabla \chi|^{2}} \operatorname{tr}\left(\nabla^{2} \chi^{T} \nabla^{2} \chi\right)\right. \\
& \left.+\left\{\operatorname{tr}\left(\nabla^{2} \chi^{T} \nabla^{2} \chi\right)-\left(\frac{\nabla \chi}{|\nabla \chi|}\right)^{T} \nabla^{2} \chi^{T} \nabla^{2} \chi\left(\frac{\nabla \chi}{|\nabla \chi|}\right)\right\}\right\} d x .
\end{aligned}
$$

Let $\nabla \chi \neq 0$. Denoting $A=\nabla^{2} \chi^{T} \nabla^{2} \chi$ we know that $A$ is a positive semi-definite matrix so we have

$$
\operatorname{tr}(A)=\sum_{i=1}^{3} \lambda_{i}(A) \geq 0
$$

where $\lambda_{i}(A)$ for $i=1,2,3$ denote the eigenvalues of $A$. Also $\left(\frac{\nabla \chi}{|\nabla \chi|}\right)^{T} A\left(\frac{\nabla \chi}{|\nabla \chi|}\right) \leq \max _{i=1,2,3} \lambda_{i}(A)$ then

$$
\operatorname{tr}(A)-\left(\frac{\nabla \chi}{|\nabla \chi|}\right)^{T} A\left(\frac{\nabla \chi}{|\nabla \chi|}\right) \geq \sum_{i=1}^{3} \lambda_{i}(A)-\max _{i=1,2,3} \lambda_{i}(A) \geq 0 .
$$

Now from (3.26) to (3.28) it follows that

$$
-\int_{\mathbb{R}^{3}} \epsilon \triangle \partial_{x_{i}} \chi \frac{\partial_{x_{i}} \chi}{\left(|\nabla \chi|^{2}+\epsilon^{2}\right)^{\frac{1}{2}}} d x \geq 0
$$

Finally by (3.23)-(3.25) and (3.29) the lemma is proved. 
Lemma 3.10. Fix $u \in L^{2}\left(0, T, E^{0}\right)$ and let $\chi=G_{\epsilon}\left(\Psi_{\epsilon}(u)\right)$ then there exists $C>0$ such that for $0<s<T$ and $\eta>0$ we have

$$
\begin{aligned}
\int_{0}^{s}\left\langle\left(J\left(f_{\epsilon}^{s . t .}(\chi)\right)\right)(t), u(t)\right\rangle d t \leq & -\int_{\mathbb{R}^{3}}\left(\left(|\nabla \chi(s)|^{2}+\epsilon^{2}\right)^{\frac{1}{2}}-\epsilon\right) d x+C\left|\nabla \chi_{0}\right|\left(\mathbb{R}^{3}\right) \\
& +\frac{C}{\eta}\left\|\chi_{0}\right\|_{L^{2}\left(\mathbb{R}^{3}\right)}^{2}+\eta\|u\|_{L^{\infty}\left(0, T, E^{0}\right)}^{2} .
\end{aligned}
$$

Proof. One may observe that for $a \in \mathbb{R}$ and $\epsilon \geq 0$ the following inequality holds

$$
\frac{a^{2}}{\left(a^{2}+\epsilon^{2}\right)^{\frac{1}{2}}} \leq 2\left(\left(a^{2}+\epsilon^{2}\right)^{\frac{1}{2}}-\epsilon\right) .
$$

Let $0<s<T$ then we have

$$
\begin{aligned}
\int_{0}^{s}\left\langle J\left(f_{\epsilon}^{s . t .}(\chi)\right)(t), u(t)\right\rangle d t= & \int_{0}^{T}\left\langle J\left(f_{\epsilon}^{s . t .}(\chi)\right)(t), 1_{(0, s)}(t) u(t)\right\rangle d t \\
= & \left\langle f_{\epsilon}^{s . t .}(\chi), 1_{(0, s)}(\cdot) u(\cdot)\right\rangle=\int_{0}^{T}\left\langle\tilde{f}_{\epsilon}^{s . t .}(\chi)(t), \Psi_{\epsilon}\left(1_{(0, s)}(\cdot) u(\cdot)\right)(t)\right\rangle d t \\
= & \int_{0}^{s}\left\langle\tilde{f}_{\epsilon}^{s . t .}(\chi)(t), \Psi_{\epsilon}(u(\cdot))(t)\right\rangle d t \\
& +\int_{s}^{\min (s+\alpha(\epsilon), T)}\left\langle\tilde{f}_{\epsilon}^{s . t .}(\chi)(t), \Psi_{\epsilon}\left(1_{(0, s)}(\cdot) u(\cdot)\right)(t)\right\rangle d t .
\end{aligned}
$$

To estimate the first term on the right hand side of (3.32) we compute using Lemma 3.9

$$
\begin{aligned}
\left\langle\tilde{f}_{\epsilon}^{s . t .}(\chi)(t), \Psi_{\epsilon}(u(\cdot))(t)\right\rangle & =\int_{\mathbb{R}^{3}} \frac{\nabla \chi \otimes \nabla \chi}{\left(|\nabla \chi|^{2}+\epsilon^{2}\right)^{\frac{1}{2}}}: \nabla \Psi_{\epsilon}(u) d x \\
& \leq-\frac{d}{d t} \int_{\mathbb{R}^{3}}\left(\left(|\nabla \chi|^{2}+\epsilon^{2}\right)^{\frac{1}{2}}-\epsilon\right) d x
\end{aligned}
$$

Now using (3.33) and (3.9) we estimate the first term on the right hand side of (3.32) as follows

$$
\begin{aligned}
\int_{0}^{s}\left\langle\tilde{f}_{\epsilon}^{s . t .}(\chi)(t), \Psi_{\epsilon}(u(\cdot))(t)\right\rangle d t & \leq-\int_{0}^{s} \frac{d}{d t}\left\{\int_{\mathbb{R}^{3}}\left(\left(|\nabla \chi|^{2}+\epsilon^{2}\right)^{\frac{1}{2}}-\epsilon\right) d x\right\} d t \\
& =-\int_{\mathbb{R}^{3}}\left(\left(|\nabla \chi(s)|^{2}+\epsilon^{2}\right)^{\frac{1}{2}}-\epsilon\right) d x+\int_{\mathbb{R}^{3}}\left(\left(\left|\nabla \chi_{0, \epsilon}\right|^{2}+\epsilon^{2}\right)^{\frac{1}{2}}-\epsilon\right) d x \\
& \leq-\int_{\mathbb{R}^{3}}\left(\left(|\nabla \chi(s)|^{2}+\epsilon^{2}\right)^{\frac{1}{2}}-\epsilon\right) d x+\int_{\mathbb{R}^{3}}\left|\nabla \chi_{0, \epsilon}\right| d x \\
& \leq-\int_{\mathbb{R}^{3}}\left(\left(|\nabla \chi(s)|^{2}+\epsilon^{2}\right)^{\frac{1}{2}}-\epsilon\right) d x+C\left|\nabla \chi_{0}\right|\left(\mathbb{R}^{3}\right) .
\end{aligned}
$$

Now we estimate the second term on the right hand side of (3.32)

$$
\begin{aligned}
& \int_{s}^{\min (s+\alpha(\epsilon), T)}\left\langle\tilde{f}_{\epsilon}^{s . t .}(\chi)(t), \Psi_{\epsilon}\left(1_{(0, s)}(\cdot) u(\cdot)\right)(t)\right\rangle d t \\
& =\int_{s}^{\min (s+\alpha(\epsilon), T)} \int_{\mathbb{R}^{3}} \frac{\nabla \chi \otimes \nabla \chi}{\left(|\nabla \chi|^{2}+\epsilon^{2}\right)^{\frac{1}{2}}}: \nabla\left(\Psi_{\epsilon}\left(1_{(0, s)}(\cdot) u(\cdot)\right)(t)\right) d x d t \\
& \leq C \int_{s}^{\min (s+\alpha(\epsilon), T)} \int_{\mathbb{R}^{3}}|\nabla \chi|\left|\nabla\left(\Psi_{\epsilon}\left(1_{(0, s)}(\cdot) u(\cdot)\right)(t)\right)\right| d x d t \\
& \leq C_{1} \frac{\epsilon}{\eta} \int_{s}^{\min (s+\alpha(\epsilon), T)} \int_{\mathbb{R}^{3}}|\nabla \chi|^{2} d x d t \\
& \quad+\frac{\eta}{\epsilon} \int_{s}^{\min (s+\alpha(\epsilon), T)} \int_{\mathbb{R}^{3}}\left|\nabla\left(\Psi_{\epsilon}\left(1_{(0, s)}(\cdot) u(\cdot)\right)(t)\right)\right|^{2} d x d t .
\end{aligned}
$$


Separately by the Lemma 3.6 and (3.8) we estimate

$$
\epsilon \int_{s}^{\min (s+\alpha(\epsilon), T)} \int_{\mathbb{R}^{3}}|\nabla \chi|^{2} d x d t \leq 4\left\|\chi_{0, \epsilon}\right\|_{L^{2}\left(\mathbb{R}^{3}\right)}^{2} \leq 4\left\|\chi_{0}\right\|_{L^{2}\left(\mathbb{R}^{3}\right)}^{2} .
$$

There exists a constant $C>0$ such that for $\gamma \in\left(L^{2}\left(\mathbb{R}^{3}\right)\right)^{3}$ and $0<\epsilon<1$

$$
\left\|\nabla\left(\tilde{\psi}_{\beta(\epsilon)} \psi_{\beta(\epsilon)} * \gamma\right)\right\|_{\left(L^{2}\left(\mathbb{R}^{3}\right)\right)^{3 \times 3}}^{2} \leq C\left(1+\frac{1}{\beta^{2}(\epsilon)}\right)\|\gamma\|_{\left(L^{2}\left(\mathbb{R}^{3}\right)\right)^{3}}^{2} .
$$

Denoting $v=\nabla\left(P \tilde{\psi}_{\beta(\epsilon)} \psi_{\beta(\epsilon)} * 1_{(0, s)}(\cdot) \bar{u}(\cdot)\right)$ and using (3.37) we estimate

$$
\begin{aligned}
\frac{1}{\epsilon} & \int_{s}^{\min (s+\alpha(\epsilon), T)} \int_{\mathbb{R}^{3}}\left|\nabla\left(\Psi_{\epsilon}\left(1_{(0, s)}(\cdot) u(\cdot)\right)(t)\right)\right|^{2} d x d t \\
& \leq \frac{1}{\epsilon} \int_{s}^{\min (s+\alpha(\epsilon), T)}\left\|\phi_{\alpha(\epsilon)} * v\right\|_{\left(L^{2}\left(\mathbb{R}^{3}\right)\right)^{3 \times 3}}^{2} d t \\
& \leq \frac{1}{\epsilon} \int_{s}^{\min (s+\alpha(\epsilon), T)}\left(\phi_{\alpha(\epsilon)} *\|v\|_{\left(L^{2}\left(\mathbb{R}^{3}\right)\right)^{3 \times 3}}\right)^{2} d t \\
& \leq \frac{1}{\epsilon} \alpha(\epsilon)\left\|\phi_{\alpha(\epsilon)} *\right\| v \|_{\left(L^{2}\left(\mathbb{R}^{3}\right)\right)^{3 \times 3} \|_{L^{\infty}(s, \min (s+\alpha(\epsilon), T))}^{2}} \\
& \leq \frac{1}{\epsilon} \alpha(\epsilon)\left\|\nabla\left(P \tilde{\psi}_{\beta(\epsilon)} \psi_{\beta(\epsilon)} * u\right)\right\|_{L^{\infty}\left((s-\alpha(\epsilon))^{+}, s,\left(L^{2}\left(\mathbb{R}^{3}\right)\right)^{3 \times 3}\right)}^{2} \\
& \leq \frac{C}{\epsilon} \alpha(\epsilon)\left(1+\frac{1}{\beta^{2}(\epsilon)}\right)\|u\|_{L^{\infty}\left((s-\alpha(\epsilon))^{+}, s,\left(L^{2}\left(\mathbb{R}^{3}\right)\right)^{3}\right)}^{2} \\
& \leq \frac{C}{\epsilon} \alpha(\epsilon)\left(1+\frac{1}{\beta^{2}(\epsilon)}\right)\|u\|_{L^{\infty}\left(0, T, E^{0}\right)}^{2} .
\end{aligned}
$$

Now by (3.35), (3.36) and (3.38) and the definitions of $\alpha(\epsilon)$ and $\beta(\epsilon)$ we obtain

$$
\int_{s}^{\min (s+\alpha(\epsilon), T)}\left\langle\tilde{f}_{\epsilon}^{s . t .}(\chi)(t), \Psi_{\epsilon}\left(1_{(0, s)}(\cdot) u(\cdot)\right)(t)\right\rangle d t \leq \frac{C_{1}}{\eta}\left\|\chi_{0}\right\|_{L^{2}\left(\mathbb{R}^{3}\right)}^{2}+C_{2} \eta\|u\|_{L^{\infty}\left(0, T, E^{0}\right)}^{2} .
$$

Finally from (3.32), (3.34) and (3.39) we obtain (3.30).

Let us consider the function $S_{\epsilon}: L^{2}\left(0, T, E^{1}\right) \rightarrow L^{2}\left(0, T, E^{1}\right)$ for $v \in L^{2}\left(0, T, E^{1}\right)$ defined by

$$
S_{\epsilon}(v)=A(w, J(f))
$$

where

$$
w=\Psi_{\epsilon}(v), \chi=G_{\epsilon}(w) \text { and } f=f_{\epsilon}^{s . t .}(\chi) .
$$

Let us note that as defined in (3.3) the operator $K_{\epsilon}$ both mollifies in space and time and localises spatially. Thus it gives the necessary compactness. Then by the continuity of $P$ we obtain that $\Psi_{\epsilon}=P K_{\epsilon}$ is compact. Further by the continuity of $G_{\epsilon}, f_{\epsilon}^{\text {s.t. }}, J$ and $A$ the mapping $S_{\epsilon}$ is compact.

Lemma 3.11. There exists a constant $C>0$ independent of $\epsilon$ such that if for some $0 \leq c \leq 1$ and $u \in L^{2}\left(0, T, E^{1}\right)$ we have $u=c S_{\epsilon}(u)$ then

$$
\begin{gathered}
c \sup _{0<s<T} \int_{\mathbb{R}^{3}}\left(\left(|\nabla \chi(s)|^{2}+\epsilon^{2}\right)^{\frac{1}{2}}-\epsilon\right) d x+\sup _{0<s<T} \int_{\mathbb{R}^{3}}|u(s)|^{2} d x \\
\quad+\int_{0}^{T} \int_{\mathbb{R}^{3}}|\nabla u|^{2} d x d t \leq C\left\{\left\|\chi_{0}\right\|_{B V\left(\mathbb{R}^{3}\right)}+\int_{\mathbb{R}^{3}}\left|u_{0}\right|^{2} d x\right\} .
\end{gathered}
$$


Proof. Let $u \in L^{2}\left(0, T, E^{1}\right), 0 \leq c \leq 1$ and $u=c S_{\epsilon}(u)$. Then testing the equation satisfied by $\frac{u}{c}$ by $u$ we get

$$
\frac{1}{2} \frac{d}{d t} \int_{\mathbb{R}^{3}}|u|^{2} d x+\int_{\mathbb{R}^{3}}|D u|^{2} d x=c\left\langle\left(J f_{\epsilon}^{s . t .}(\chi)\right)(t), u\right\rangle
$$

we then integrate in time on $(0, s)$ for some $0<s<T$ and by Lemma 3.10, the inequality $c \leq 1$ and small $\eta>0$ we obtain

$$
\begin{aligned}
& c \int_{\mathbb{R}^{3}}\left(\left(|\nabla \chi(s)|^{2}+\epsilon^{2}\right)^{\frac{1}{2}}-\epsilon\right) d x+\int_{\mathbb{R}^{3}}|u(s)|^{2} d x+\frac{1}{2} \int_{0}^{s} \int_{\mathbb{R}^{3}}|\nabla u|^{2} d x d t \\
& \leq \int_{\mathbb{R}^{3}}\left|u_{0}\right|^{2} d x+C\left|\nabla \chi_{0}\right|\left(\mathbb{R}^{3}\right)+\frac{C}{\eta}\left\|\chi_{0}\right\|_{L^{2}\left(\mathbb{R}^{3}\right)}^{2}+\eta\|u\|_{L^{\infty}\left(0, T, E^{0}\right)}^{2} .
\end{aligned}
$$

And from this by choosing $\eta>0$ small enough it follows that

$$
\begin{aligned}
& c \sup _{0<s<T} \int_{\mathbb{R}^{3}}\left(\left(|\nabla \chi(s)|^{2}+\epsilon^{2}\right)^{\frac{1}{2}}-\epsilon\right) d x+\sup _{0<s<T} \int_{\mathbb{R}^{3}}|u(s)|^{2} d x \\
& \quad+\int_{0}^{T} \int_{\mathbb{R}^{3}}|\nabla u|^{2} d x d t \leq C_{1}\left\{\int_{\mathbb{R}^{3}}\left|u_{0}\right|^{2} d x+\left|\nabla \chi_{0}\right|\left(\mathbb{R}^{3}\right)+\left\|\chi_{0}\right\|_{L^{2}\left(\mathbb{R}^{3}\right)}^{2}\right\}
\end{aligned}
$$

which together with $0 \leq \chi_{0} \leq 1$ proves $(3.40)$.

Let us define

$$
E_{a . s .}^{1}=\text { axisymmetric functions in } E^{1} .
$$

It is clear that $E_{a . s .}^{1}$ is a closed subspace of $E^{1}$.

Theorem 3.12. There exists $u_{\epsilon} \in L^{2}\left(0, T, E^{1}\right)$ solution to the system of Eqs. (3.10) and (3.11). If the initial values $u_{0}$ and $\Omega_{1,0}$ are axisymmetric then there exists $u_{\epsilon} \in L^{2}\left(0, T, E_{\text {a.s. }}^{1}\right)$ solution to the system of Eqs. (3.10) and (3.11).

Proof. By Lemma 3.4 the map $\Psi_{\epsilon}: L^{2}\left(0, T, E^{1}\right) \rightarrow W$ is compact [we consider the $\left(C_{b}\left(\mathbb{R}^{3}\right)\right)^{3}$ norm for $W]$, by Lemma 3.6 the map $G_{\epsilon}: W \rightarrow L^{2}\left(0, T, H^{1}\left(\mathbb{R}^{3}\right)\right)$ is continuous, by Lemma 3.7 the force term

$$
f_{\epsilon}^{\text {s.t. }:} L^{2}\left(0, T, H^{1}\left(\mathbb{R}^{3}\right)\right) \rightarrow\left(L^{2}\left(0, T,\left(L^{2}\left(\mathbb{R}^{3}\right)\right)^{3}\right)\right)^{*}
$$

is continuous, we know that

$$
J:\left(L^{2}\left(0, T,\left(L^{2}\left(\mathbb{R}^{3}\right)\right)^{3}\right)\right)^{*} \rightarrow L^{2}\left(0, T,\left(\left(L^{2}\left(\mathbb{R}^{3}\right)\right)^{3}\right)^{*}\right)
$$

is continuous and by Lemma 3.8 the map $A: W \times L^{2}\left(0, T,\left(\left(L^{2}\left(\mathbb{R}^{3}\right)\right)^{3}\right)^{*}\right) \rightarrow L^{2}\left(0, T, E^{1}\right)$ is continuous. Thus the map $S_{\epsilon}$ is compact and in particular continuous. By the previous lemma we have that the set

$$
X_{\epsilon}=\left\{v \in L^{2}\left(0, T, E^{1}\right) \mid v=c S_{\epsilon}(v) \text { for some } 0 \leq c \leq 1\right\}
$$

is bounded and hence we might apply Leray-Schauder principle (cf. Schaefer's fixed point theorem in [7]) to obtain a fixed point $u_{\epsilon} \in L^{2}\left(0, T, E^{1}\right), u_{\epsilon}=S_{\epsilon}\left(u_{\epsilon}\right)$ which proves the existence of a solution.

Now let us consider the case when the initial values $u_{0}$ and $\Omega_{1,0}$ are axisymmetric. For $v \in L^{2}\left(0, T, E_{\text {a.s. }}^{1}\right)$ by the commuting properties of $\Psi_{\epsilon}$ as discussed in the Lemma 3.4 we have that $w=\Psi_{\epsilon}(v)$ is axisymmetric, by the Lemma 3.6 we have that $\chi$ is axisymmetric, by Lemma 3.7 the force term $f=f_{\epsilon}^{\text {s.t. }}(\chi)$ is axisymmetric, it is easy to see that $J(f)$ is axisymmetric and finally by Lemma 3.8 we have that $S_{\epsilon}(v)$ is axisymmetric. Thus we have $S_{\epsilon}: L^{2}\left(0, T, E_{a . s .}^{1}\right) \rightarrow L^{2}\left(0, T, E_{a . s .}^{1}\right)$. Now because

$$
X_{\epsilon, a . s .}=\left\{v \in L^{2}\left(0, T, E_{a . s .}^{1}\right) \mid v=c S_{\epsilon}(v) \text { for some } 0 \leq c \leq 1\right\} \subset X_{\epsilon},
$$

the set $X_{\epsilon, a . s}$. is bounded and we might apply Leray-Schauder principle to obtain a fixed point $u_{\epsilon} \in$ $L^{2}\left(0, T, E_{a . s .}^{1}\right), u_{\epsilon}=S_{\epsilon}\left(u_{\epsilon}\right)$ which proves the existence of an axisymmetric solution. 


\section{Existence of Varifold Solution}

In the following for each $0<\epsilon<1$ we consider the pair $u_{\epsilon} \in L^{2}\left(0, T, E^{1}\right)$ and $\chi_{\epsilon} \in L^{2}\left(0, T, H^{1}\left(\mathbb{R}^{3}\right)\right)$ a solution to the system of Eqs. (3.10) and (3.11). The existence of these is proved in the Theorem 3.12.

\subsection{Uniform Bounds on time Derivatives}

Lemma 4.1. We have $\sup _{0<\epsilon<1}\left\|\partial_{t} \chi_{\epsilon}\right\|_{L^{2}\left(0, T, H^{-1}\left(\mathbb{R}^{3}\right)\right)}<\infty$.

Proof. We have

$$
\partial_{t} \chi_{\epsilon}=-\Psi_{\epsilon}\left(u_{\epsilon}\right) \cdot \nabla \chi_{\epsilon}+\epsilon \triangle \chi_{\epsilon}
$$

in $H^{-1}\left(\mathbb{R}^{3}\right)$ for a.e. $t>0$.

For the first term on the right hand side of (4.1) we compute for $\varphi \in H^{1}\left(\mathbb{R}^{3}\right)$ by (3.7)

$$
\begin{aligned}
\left\langle-\Psi_{\epsilon}\left(u_{\epsilon}\right) \cdot \nabla \chi_{\epsilon}, \varphi\right\rangle & =-\int_{\mathbb{R}^{3}} \Psi_{\epsilon}\left(u_{\epsilon}\right) \cdot \nabla \chi_{\epsilon} \varphi d x=\int_{\mathbb{R}^{3}} \Psi_{\epsilon}\left(u_{\epsilon}\right) \cdot \nabla \varphi \chi_{\epsilon} d x \\
& \leq \int_{\mathbb{R}^{3}}\left|\Psi_{\epsilon}\left(u_{\epsilon}\right)\right||\nabla \varphi| d x \leq\left\|\Psi_{\epsilon}\left(u_{\epsilon}\right)\right\|_{L^{2}\left(\mathbb{R}^{3}\right)}\|\varphi\|_{H^{1}\left(\mathbb{R}^{3}\right)}
\end{aligned}
$$

thus we have

$$
\left\|-\Psi_{\epsilon}\left(u_{\epsilon}\right) \cdot \nabla \chi_{\epsilon}\right\|_{L^{2}\left(0, T, H^{-1}\left(\mathbb{R}^{3}\right)\right)}^{2} \leq \int_{0}^{T}\left\|\Psi_{\epsilon}\left(u_{\epsilon}\right)\right\|_{\left(L^{2}\left(\mathbb{R}^{3}\right)\right)^{3}}^{2} d t \leq C \int_{0}^{T}\left\|u_{\epsilon}\right\|_{\left(L^{2}\left(\mathbb{R}^{3}\right)\right)^{3}}^{2} d t .
$$

For the second term on the right hand side of (4.1) we have for $\varphi \in H^{1}\left(\mathbb{R}^{3}\right)$

$$
\left\langle\epsilon \triangle \chi_{\epsilon}, \varphi\right\rangle=\epsilon \int_{\mathbb{R}^{3}} \triangle \chi_{\epsilon} \varphi d x=-\epsilon \int_{\mathbb{R}^{3}} \nabla \chi_{\epsilon} \cdot \nabla \varphi d x \leq \epsilon\left\{\int_{\mathbb{R}^{3}}\left|\nabla \chi_{\epsilon}\right|^{2} d x\right\}^{\frac{1}{2}}\|\varphi\|_{H^{1}\left(\mathbb{R}^{3}\right)}
$$

thus by Lemma 3.6 and (3.8) we have

$$
\left\|\epsilon \triangle \chi_{\epsilon}\right\|_{L^{2}\left(0, T, H^{-1}\left(\mathbb{R}^{3}\right)\right)}^{2} \leq \epsilon^{2} \int_{0}^{T} \int_{\mathbb{R}^{3}}\left|\nabla \chi_{\epsilon}\right|^{2} d x d t \leq C\left\|\chi_{0}\right\|_{L^{2}\left(\mathbb{R}^{3}\right)}^{2} .
$$

Now by (4.1)-(4.3) and Lemma 3.11 the lemma is proved.

Let

$$
E^{3}=\text { closure of }\left\{v \in\left(C_{c}^{\infty}\left(\mathbb{R}^{3}\right)\right)^{3} \mid \operatorname{div}(v)=0\right\} \quad \text { in }\left(H^{3}\left(\mathbb{R}^{3}\right)\right)^{3} .
$$

Lemma 4.2. We have $\sup _{0<\epsilon<1}\left\|\partial_{t} u_{\epsilon}\right\|_{L^{1}\left(0, T,\left(E^{3}\right)^{*}\right)}<\infty$.

Proof. By the equation satisfied by $u_{\epsilon}$ we have

$$
\partial_{t} u_{\epsilon}=-\operatorname{div}\left(\Psi_{\epsilon}\left(u_{\epsilon}\right) \otimes u_{\epsilon}\right)+\operatorname{div}\left(D\left(u_{\epsilon}\right)\right)+J\left(f_{\epsilon}^{s . t .}\left(\chi_{\epsilon}\right)\right)
$$

in $\left(E^{1}\right)^{*}$ for a.e. $0<t<T$.

By Sobolev inequality in $\mathbb{R}^{3}$, for $u \in H^{1}\left(\mathbb{R}^{3}\right)$ we have $\|u\|_{L^{6}\left(\mathbb{R}^{3}\right)} \leq C\|\nabla u\|_{\left(L^{2}\left(\mathbb{R}^{3}\right)\right)^{3}}$ from here it follows that $\|u\|_{L^{4}\left(\mathbb{R}^{3}\right)} \leq C_{1}\|u\|_{H^{1}\left(\mathbb{R}^{3}\right)}$. Now for the first term on the right hand side of (4.4) we have for $\varphi \in E^{3}$

$$
\begin{aligned}
\left\langle-\operatorname{div}\left(\Psi_{\epsilon}\left(u_{\epsilon}\right) \otimes u_{\epsilon}\right), \varphi\right\rangle_{\left(E^{1}\right)^{*}, E^{1}} & =\int_{\mathbb{R}^{3}}\left(\Psi_{\epsilon}\left(u_{\epsilon}\right)\right)_{i}\left(u_{\epsilon}\right)_{j} \partial_{x_{i}} \varphi_{j} d x \\
& \leq C_{1} \int_{\mathbb{R}^{3}}\left|\Psi_{\epsilon}\left(u_{\epsilon}\right)\right|\left|u_{\epsilon}\right||\nabla \varphi| d x \\
& \leq C_{1}\left\{\int_{\mathbb{R}^{3}}\left|\Psi_{\epsilon}\left(u_{\epsilon}\right)\right|^{4} d x\right\}^{\frac{1}{4}}\left\{\int_{\mathbb{R}^{3}}\left|u_{\epsilon}\right|^{4} d x\right\}^{\frac{1}{4}}\left\{\int_{\mathbb{R}^{3}}|\nabla \varphi|^{2} d x\right\}^{\frac{1}{2}} \\
& \leq C_{2}\left\|\Psi_{\epsilon}\left(u_{\epsilon}\right)\right\|_{\left(H^{1}\left(\mathbb{R}^{3}\right)\right)^{3}}\left\|u_{\epsilon}\right\|_{\left(H^{1}\left(\mathbb{R}^{3}\right)\right)^{3}}\|\varphi\|_{E^{3}} .
\end{aligned}
$$


One may check that there exists a constant $C>0$ such that for $v \in L^{2}\left(0, T,\left(H^{1}\left(\mathbb{R}^{3}\right)\right)^{3}\right)$ we have

$$
\int_{0}^{T}\left\|\Psi_{\epsilon}(v)\right\|_{\left(H^{1}\left(\mathbb{R}^{3}\right)\right)^{3}}^{2} d t \leq C \int_{0}^{T}\|v\|_{\left(H^{1}\left(\mathbb{R}^{3}\right)\right)^{3}}^{2} d t
$$

thus

$$
\begin{aligned}
\left\|-\operatorname{div}\left(\Psi_{\epsilon}\left(u_{\epsilon}\right) \otimes u_{\epsilon}\right)\right\|_{L^{1}\left(0, T,\left(E^{3}\right)^{*}\right)} & \leq C_{2}\left\{\int_{0}^{T}\left\|\Psi_{\epsilon}\left(u_{\epsilon}\right)\right\|_{\left(H^{1}\left(\mathbb{R}^{3}\right)\right)^{3}}^{2} d t+\int_{0}^{T}\left\|u_{\epsilon}\right\|_{\left(H^{1}\left(\mathbb{R}^{3}\right)\right)^{3}}^{2} d t\right\} \\
& \leq C_{3} \int_{0}^{T}\left\|u_{\epsilon}\right\|_{\left(H^{1}\left(\mathbb{R}^{3}\right)\right)^{3}}^{2} d t .
\end{aligned}
$$

By the Sobolev inequality there exists a constant $C>0$ such that

$$
\|v\|_{C_{b}^{1}\left(\mathbb{R}^{3}\right)} \leq C\|v\|_{H^{3}\left(\mathbb{R}^{3}\right)} \text { for all } v \in H^{3}\left(\mathbb{R}^{3}\right) .
$$

For the second term on the right hand side of (4.4) we have for $\varphi \in E^{3}$

$$
\left\langle\operatorname{div}\left(D\left(u_{\epsilon}\right)\right), \varphi\right\rangle_{\left(E^{1}\right)^{*}, E^{1}}=-\int_{\mathbb{R}^{3}} D u_{\epsilon}: D \varphi d x \leq C\left\{\int_{\mathbb{R}^{3}}\left|\nabla u_{\epsilon}\right|^{2} d x\right\}^{\frac{1}{2}}\|\varphi\|_{E^{3}}
$$

thus

$$
\left\|\operatorname{div}\left(D u_{\epsilon}\right)\right\|_{L^{1}\left(0, T,\left(E^{3}\right)^{*}\right)} \leq C \int_{0}^{T}\left\{\int_{\mathbb{R}^{3}}\left|\nabla u_{\epsilon}\right|^{2} d x\right\}^{\frac{1}{2}} d t \leq C T^{\frac{1}{2}}\left\{\int_{0}^{T}\left\|u_{\epsilon}\right\|_{\left(H^{1}\left(\mathbb{R}^{3}\right)\right)^{3}}^{2} d t\right\}^{\frac{1}{2}}
$$

For the third term on the right hand side of (4.4) we have for $\varphi \in\left(C_{c}^{\infty}\left(\mathbb{R}^{4}\right)\right)^{3}$

$$
\begin{aligned}
\int_{0}^{T}\left\langle J\left(f_{\epsilon}^{\text {s.t. }}\left(\chi_{\epsilon}\right)\right)(t), \varphi\right\rangle d t & =\left\langle f_{\epsilon}^{\text {s.t. }}\left(\chi_{\epsilon}\right), \varphi\right\rangle=\int_{0}^{T}\left\langle\tilde{f}_{\epsilon}^{\text {s.t. }}\left(\chi_{\epsilon}(t)\right), \Psi_{\epsilon}(\varphi)\right\rangle d t \\
& =\int_{0}^{T} \int_{\mathbb{R}^{3}} \frac{\nabla \chi_{\epsilon} \otimes \nabla \chi_{\epsilon}}{\left(\left|\nabla \chi_{\epsilon}\right|^{2}+\epsilon^{2}\right)^{\frac{1}{2}}}: \nabla \Psi_{\epsilon}(\varphi) d x d t \\
& \leq C_{1} \int_{0}^{T} \int_{\mathbb{R}^{3}} \frac{\left|\nabla \chi_{\epsilon}\right|^{2}}{\left(\left|\nabla \chi_{\epsilon}\right|^{2}+\epsilon^{2}\right)^{\frac{1}{2}}}\left|\nabla \Psi_{\epsilon}(\varphi)\right| d x d t \\
& \leq C_{2} \int_{0}^{T} \int_{\mathbb{R}^{3}}\left(\left(\left|\nabla \chi_{\epsilon}\right|^{2}+\epsilon^{2}\right)^{\frac{1}{2}}-\epsilon\right)\left|\nabla \Psi_{\epsilon}(\varphi)\right| d x d t \\
& \leq C_{2}\left\{\sup _{0<t<T} \int_{\mathbb{R}^{3}}\left(\left(\left|\nabla \chi_{\epsilon}\right|^{2}+\epsilon^{2}\right)^{\frac{1}{2}}-\epsilon\right) d x\right\} \int_{0}^{T}\left\|\nabla \Psi_{\epsilon}(\varphi)\right\|_{\left(C\left(\mathbb{R}^{3}\right)\right)^{3 \times 3}} d t .
\end{aligned}
$$

denoting $v=\nabla P\left(\tilde{\psi}_{\beta(\epsilon)} \psi_{\beta(\epsilon)} * \bar{\varphi}\right)$ using the Minkowski inequality once and then the Sobolev inequality (4.6), separately we estimate

$$
\begin{aligned}
\int_{0}^{T}\left\|\nabla \Psi_{\epsilon}(\varphi)\right\|_{\left(C\left(\mathbb{R}^{3}\right)\right)^{3 \times 3}} d t & \leq \int_{0}^{T} \phi_{\alpha(\epsilon)} *\|v\|_{\left(C\left(\mathbb{R}^{3}\right)\right)^{3 \times 3}} d t \\
& \leq \int_{0}^{T}\|v\|_{\left(C\left(\mathbb{R}^{3}\right)\right)^{3 \times 3}} d t \leq C_{1} \int_{0}^{T}\left\|P\left(\tilde{\psi}_{\beta(\epsilon)} \psi_{\beta(\epsilon)} * \varphi\right)\right\|_{\left(H^{3}\left(\mathbb{R}^{3}\right)\right)^{3}} d t \\
& \leq C_{2} \int_{0}^{T}\left\|\tilde{\psi}_{\beta(\epsilon)} \psi_{\beta(\epsilon)} * \varphi\right\|_{\left(H^{3}\left(\mathbb{R}^{3}\right)\right)^{3}} d t \leq C_{3}\|\varphi\|_{L^{1}\left(0, T,\left(H^{3}\left(\mathbb{R}^{3}\right)\right)^{3}\right)}
\end{aligned}
$$

so we have

$$
\int_{0}^{T}\left\langle J\left(f_{\epsilon}^{s . t .}\left(\chi_{\epsilon}\right)\right)(t), \varphi\right\rangle d t \leq C\left\{\sup _{0<t<T} \int_{\mathbb{R}^{3}}\left(\left(\left|\nabla \chi_{\epsilon}\right|^{2}+\epsilon^{2}\right)^{\frac{1}{2}}-\epsilon\right) d x\right\}\|\varphi\|_{L^{1}\left(0, T, E^{3}\right)} .
$$


As mentioned in Sect. 2, because $\left(E^{1}\right)^{*}$ is a Hilbert space it is in particular a reflexive Banach space and hence $L^{\infty}\left(0, T,\left(E^{3}\right)^{*}\right)=\left(L^{1}\left(0, T, E^{3}\right)\right)^{*}$ so we have

$$
\begin{aligned}
\left\|J\left(f_{\epsilon}^{s . t .}\left(\chi_{\epsilon}\right)\right)\right\|_{L^{1}\left(0, T,\left(E^{3}\right)^{*}\right)} & \leq T\left\|J\left(f_{\epsilon}^{s . t .}\left(\chi_{\epsilon}\right)\right)\right\|_{L^{\infty}\left(0, T,\left(E^{3}\right)^{*}\right)} \\
& \leq C_{1} T\left\|J\left(f_{\epsilon}^{s . t .}\left(\chi_{\epsilon}\right)\right)\right\|_{\left(L^{1}\left(0, T, E^{3}\right)\right)^{*}} \\
& \leq C_{2} T \sup _{0<t<T} \int_{\mathbb{R}^{3}}\left(\left(\left|\nabla \chi_{\epsilon}\right|^{2}+\epsilon^{2}\right)^{\frac{1}{2}}-\epsilon\right) d x .
\end{aligned}
$$

Now by (4.4), (4.5), (4.7), (4.8) and Lemma 3.11 the lemma is proved.

\section{2. $w^{*}$ Compactness for Measure Valued Functions}

Lemma 4.3. Let for some $\Lambda>0$

$$
\left\|\nu_{k}\right\|_{L_{w^{*}}^{\infty}\left(0, T, \mathcal{M}\left(\mathbb{R}^{d}\right)\right)} \leq \Lambda, \quad \forall k \in \mathbb{N}
$$

then there exists a subsequence $k_{n}$ and $\nu \in L_{w^{*}}^{\infty}\left(0, T, \mathcal{M}\left(\mathbb{R}^{d}\right)\right)$ such that

$$
\int_{0}^{T} \int_{\mathbb{R}^{d}} \varphi \nu_{k_{n}}(t)(d x) d t \rightarrow \int_{0}^{T} \int_{\mathbb{R}^{d}} \varphi(t, x) \nu(t)(d x) d t, \quad \forall \varphi \in L^{1}\left(0, T, C_{0}\left(\mathbb{R}^{d}\right)\right)
$$

and

$$
\|\nu\|_{L_{w^{*}}^{\infty}\left(0, T, \mathcal{M}\left(\mathbb{R}^{d}\right)\right)} \leq \liminf _{k \rightarrow \infty}\left\|\nu_{k}\right\|_{L_{w^{*}}^{\infty}\left(0, T, \mathcal{M}\left(\mathbb{R}^{d}\right)\right)}
$$

Proof. Step 1. If $m \in \mathbb{N}$ such that $m>\frac{d}{2}$ then it follows from Sobolev embedding theorem that we have the continuous, dense and injective embedding

$$
H^{m}\left(\mathbb{R}^{d}\right) \hookrightarrow C_{0}\left(\mathbb{R}^{d}\right)
$$

and by the Ritz representation theorem it follows the continuous, dense and injective embedding

$$
\mathcal{M}\left(\mathbb{R}^{d}\right)=\left(C_{0}\left(\mathbb{R}^{d}\right)\right)^{*} \hookrightarrow H^{-m}\left(\mathbb{R}^{d}\right) .
$$

Step 2. In this step our aim is to show the continuous embedding

$$
L_{w^{*}}^{\infty}\left(0, T, \mathcal{M}\left(\mathbb{R}^{d}\right)\right) \hookrightarrow L_{w^{*}}^{\infty}\left(0, T, H^{-m}\left(\mathbb{R}^{d}\right)\right)
$$

holds.

Let $\gamma \in L_{w^{*}}^{\infty}\left(0, T, \mathcal{M}\left(\mathbb{R}^{d}\right)\right)$. By $(4.12)$ we have that $\gamma:(0, T) \rightarrow H^{-3}\left(\mathbb{R}^{d}\right)$.

To show that $\gamma$ with values in $H^{-3}\left(\mathbb{R}^{d}\right)$ is $w^{*} \lambda$-measurable let $\phi \in H^{m}\left(\mathbb{R}^{d}\right)$ then

$$
\langle\gamma(t), \phi\rangle_{H^{-m}\left(\mathbb{R}^{d}\right), H^{m}\left(\mathbb{R}^{d}\right)}=\langle\gamma(t), \phi\rangle_{\mathcal{M}\left(\mathbb{R}^{d}\right), C_{0}\left(\mathbb{R}^{d}\right)}
$$

and because $\gamma$ with values in $\mathcal{M}\left(\mathbb{R}^{d}\right)$ is $w^{*} \lambda$-measurable the function on the right hand side of (4.14) is measurable and hence so is the function on the left hand side, which proves the desired property.

Now from the separability of $H^{m}\left(\mathbb{R}^{d}\right)$ it follows that $\|\gamma(t)\|_{H^{-m}\left(\mathbb{R}^{d}\right)}$ as a function of $t$ is measurable.

Finally by (4.12) we have that for a.e. $0<t<T$ we have

$$
\|\gamma(t)\|_{H^{-m}\left(\mathbb{R}^{d}\right)} \leq C\|\gamma(t)\|_{\mathcal{M}\left(\mathbb{R}^{d}\right)} \leq C\|\gamma\|_{L_{w^{*}}^{\infty}\left(0, T, \mathcal{M}\left(\mathbb{R}^{d}\right)\right)}
$$

which shows that $\|\gamma\|_{L_{w^{*}}^{\infty}\left(0, T, H^{-m}\left(\mathbb{R}^{d}\right)\right)} \leq C\|\gamma\|_{L_{w^{*}}^{\infty}\left(0, T, \mathcal{M}\left(\mathbb{R}^{d}\right)\right)}$ and this proves (4.13).

Step 3. In this step our aim is to show that

$$
L_{w^{*}}^{\infty}\left(0, T, H^{-m}\left(\mathbb{R}^{d}\right)\right)=L^{\infty}\left(0, T, H^{-m}\left(\mathbb{R}^{d}\right)\right)
$$

It is obvious that by the identity map $L^{\infty}\left(0, T, H^{-m}\left(\mathbb{R}^{d}\right)\right) \hookrightarrow L_{w^{*}}^{\infty}\left(0, T, H^{-m}\left(\mathbb{R}^{d}\right)\right)$ continuously as $\lambda$-measurability is stronger than $w^{*} \lambda$-measurability. So it remains to show that by the identity map we have the continuous embedding

$$
L_{w^{*}}^{\infty}\left(0, T, H^{-m}\left(\mathbb{R}^{d}\right)\right) \hookrightarrow L^{\infty}\left(0, T, H^{-m}\left(\mathbb{R}^{d}\right)\right) .
$$


Now let $\gamma \in L_{w^{*}}^{\infty}\left(0, T, H^{-m}\left(\mathbb{R}^{d}\right)\right)$ and we should show that $\gamma$ is $\lambda$-measurable. Because $H^{-m}\left(\mathbb{R}^{d}\right)$ is separable we have that $\gamma$ is $\lambda$-essentially separably valued. Because $H^{m}\left(\mathbb{R}^{d}\right)$ is a Hilbert space in particular it is a reflexive space and hence by $w^{*} \lambda$-measurability of $\gamma, \gamma$ is weakly $\lambda$-measurable. Hence by Pettis measurability theorem $\gamma$ is $\lambda$-measurable and this proves (4.16) which in turn proves (4.15).

Step 4. In this step our aim is to obtain a $w^{*}$ convergent subsequence of $\nu_{k}$ in $L^{\infty}\left(0, T, H^{-m}\left(\mathbb{R}^{d}\right)\right)$.

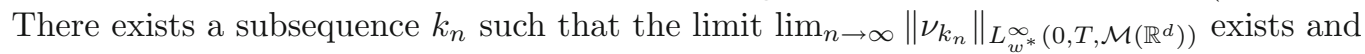

$$
\lim _{n \rightarrow \infty}\left\|\nu_{k_{n}}\right\|_{L_{w^{*}}^{\infty}\left(0, T, \mathcal{M}\left(\mathbb{R}^{d}\right)\right)}=\liminf _{k \rightarrow \infty}\left\|\nu_{k}\right\|_{L_{w^{*}}^{\infty}\left(0, T, \mathcal{M}\left(\mathbb{R}^{d}\right)\right)} .
$$

In the following for ease of notation let us denote

$$
\Lambda^{\prime}=\liminf _{k \rightarrow \infty}\left\|\nu_{k}\right\|_{L_{w^{*}}^{\infty}\left(0, T, \mathcal{M}\left(\mathbb{R}^{d}\right)\right)}
$$

Because $H^{-m}\left(\mathbb{R}^{d}\right)$ is a Hilbert space we have

$$
L^{\infty}\left(0, T, H^{-m}\left(\mathbb{R}^{d}\right)\right)=\left(L^{1}\left(0, T, H^{m}\left(\mathbb{R}^{d}\right)\right)\right)^{*}
$$

(see Sect. 2).

By (4.9) and (4.13) we have that $\nu_{k_{n}}$ is uniformly bounded in $L_{w^{*}}^{\infty}\left(0, T, H^{-m}\left(\mathbb{R}^{d}\right)\right)$. By the previous step we obtain that $\nu_{k}$ is uniformly bounded in $L^{\infty}\left(0, T, H^{-m}\left(\mathbb{R}^{d}\right)\right)$. Now by Anaoglu theorem there exists a $\nu \in L^{\infty}\left(0, T, H^{-m}\left(\mathbb{R}^{d}\right)\right)$ and a subsequence $k_{n_{\ell}}$ such that $\nu_{k_{n_{\ell}}}, w^{*}$ converges to $\nu$ in $L^{\infty}\left(0, T, H^{-m}\left(\mathbb{R}^{d}\right)\right)$, i.e.

$$
\int_{0}^{T}\left\langle\nu_{k_{n_{\ell}}}(t), \phi(t)\right\rangle_{H^{-m}\left(\mathbb{R}^{d}\right), H^{m}\left(\mathbb{R}^{d}\right)} d t \rightarrow \int_{0}^{T}\langle\nu(t), \phi(t)\rangle_{H^{-m}\left(\mathbb{R}^{d}\right), H^{m}\left(\mathbb{R}^{d}\right)} d t
$$

for all $\phi \in L^{1}\left(0, T, H^{m}\left(\mathbb{R}^{d}\right)\right)$ as $\ell \rightarrow \infty$.

Step 5. In this step our aim is to show that $\nu \in L_{w^{*}}^{\infty}\left(0, T, \mathcal{M}\left(\mathbb{R}^{d}\right)\right)$ with

$$
\|\nu\|_{L_{w^{*}}^{\infty}\left(0, T, \mathcal{M}\left(\mathbb{R}^{d}\right)\right)} \leq \Lambda^{\prime} .
$$

Let $\phi \in L^{1}\left(0, T, H^{m}\left(\mathbb{R}^{d}\right)\right)$ then by the uniform bound (4.9) we have that

$$
\begin{aligned}
\int_{0}^{T}\left\langle\nu_{k_{n_{\ell}}}(t), \phi(t)\right\rangle_{H^{-m}\left(\mathbb{R}^{d}\right), H^{m}\left(\mathbb{R}^{d}\right)} d t & =\int_{0}^{T}\left\langle\nu_{k_{n_{\ell}}}(t), \phi(t)\right\rangle_{\mathcal{M}\left(\mathbb{R}^{d}\right), C_{0}\left(\mathbb{R}^{d}\right)} d t \\
& \leq\left\|\nu_{k_{n_{\ell}}}\right\|_{L_{w^{*}}^{\infty}\left(0, T, \mathcal{M}\left(\mathbb{R}^{d}\right)\right)}\|\phi\|_{L^{1}\left(0, T, C_{0}\left(\mathbb{R}^{d}\right)\right)}
\end{aligned}
$$

by (4.17) and (4.18) passing to the limit in the inequality above we obtain

$$
\int_{0}^{T}\langle\nu(t), \phi(t)\rangle_{H^{-m}\left(\mathbb{R}^{d}\right), H^{m}\left(\mathbb{R}^{d}\right)} d t \leq \Lambda^{\prime}\|\phi\|_{L^{1}\left(0, T, C_{0}\left(\mathbb{R}^{d}\right)\right)}
$$

and by Lebesgue differentiation theorem we obtain that

$$
\langle\nu(t), \varphi\rangle_{H^{-m}\left(\mathbb{R}^{d}\right), H^{m}\left(\mathbb{R}^{d}\right)} \leq \Lambda^{\prime}\|\varphi\|_{C_{0}\left(\mathbb{R}^{d}\right)} \quad \text { for a.e. } t \in(0, T) \text { and } \varphi \in H^{m}\left(\mathbb{R}^{d}\right) .
$$

From (4.20) and the density of the embedding (4.11) it follows that $\nu(t) \in \mathcal{M}\left(\mathbb{R}^{d}\right)$ for a.e. $t \in(0, T)$ and

$$
\|\nu(t)\|_{\mathcal{M}\left(\mathbb{R}^{d}\right)} \leq \Lambda^{\prime} \quad \text { for a.e. } t \in(0, T) .
$$

Now let us show that $\nu$ with a.e. values in $\mathcal{M}\left(\mathbb{R}^{d}\right)$ is $w^{*} \lambda$-measurable. Let $\varphi \in C_{0}\left(\mathbb{R}^{d}\right)$ and $\varphi_{n} \in$ $H^{m}\left(\mathbb{R}^{d}\right)$ such that $\varphi_{n} \rightarrow \varphi$ in $C_{0}\left(\mathbb{R}^{d}\right)$. Then we have

$$
\langle\nu(t), \varphi\rangle_{\mathcal{M}\left(\mathbb{R}^{d}\right), C_{0}\left(\mathbb{R}^{d}\right)}=\lim _{n \rightarrow \infty}\left\langle\nu(t), \varphi_{n}\right\rangle_{H^{-m}\left(\mathbb{R}^{d}\right), H^{m}\left(\mathbb{R}^{d}\right)}
$$

and because $\nu \in L^{\infty}\left(0, T, H^{-m}\left(\mathbb{R}^{d}\right)\right)$ and $\varphi_{n} \in H^{m}\left(\mathbb{R}^{d}\right)$ each of the functions (as function of $t$ ) on the right hand side is measurable and thus left hand side as the limit of a sequence is measurable. 
From separability of $C_{0}\left(\mathbb{R}^{d}\right)$ it follows that $\|\nu(t)\|_{\mathcal{M}\left(\mathbb{R}^{d}\right)}$ as a function of $t$ is measurable. These measurability results together with (4.21) prove (4.19).

Step 6. In this step our aim is to prove the continuous and dense embedding

$$
L^{1}\left(0, T, H^{m}\left(\mathbb{R}^{d}\right)\right) \hookrightarrow L^{1}\left(0, T, C_{0}\left(\mathbb{R}^{d}\right)\right) .
$$

Let $\phi \in L^{1}\left(0, T, H^{m}\left(\mathbb{R}^{d}\right)\right)$ then by the embedding (4.11) we have that $\phi:(0, T) \rightarrow C_{0}\left(\mathbb{R}^{d}\right)$. By the $\lambda$-measurability of $\phi$ with values in $H^{m}\left(\mathbb{R}^{d}\right)$ there exists a sequence of simple functions $s_{n}$ with values in $H^{m}\left(\mathbb{R}^{d}\right)$ such that $\left\|s_{n}(t)-\phi(t)\right\|_{H^{m}\left(\mathbb{R}^{d}\right)} \rightarrow 0$ for a.e. $t \in(0, T)$ hence by $(4.11)$, also $\left\|s_{n}(t)-\phi(t)\right\|_{C_{0}\left(\mathbb{R}^{d}\right)} \rightarrow$ 0 for a.e. $t \in(0, T)$. Thus $\phi$ is also $\lambda$-measurable with values in $C_{0}\left(\mathbb{R}^{d}\right)$.

By the embedding (4.11) it is easy to see that the embedding (4.22) is continuous.

Now let us show the density of the embedding (4.22).

Let $\phi \in L^{1}\left(0, T, C_{0}\left(\mathbb{R}^{d}\right)\right)$. Let us show that there exists a sequence of simple functions $s_{k} \in L^{1}\left(0, T, H^{m}\right.$ $\left.\left(\mathbb{R}^{d}\right)\right)$ such that $s_{k} \rightarrow \phi$ in $L^{1}\left(0, T, C_{0}\left(\mathbb{R}^{d}\right)\right)$.

By the separability of $H^{m}\left(\mathbb{R}^{d}\right)$ and its density by $(4.11)$ in $C_{0}\left(\mathbb{R}^{d}\right)$ there exists a countable sequence $g_{n} \in H^{m}\left(\mathbb{R}^{d}\right)$ which is dense in $C_{0}\left(\mathbb{R}^{d}\right)$.

Let us define for $k, n \in \mathbb{N}$

$$
E_{k, n}=\left\{t \in(0, T) \mid\left\|\phi(t)-g_{n}\right\|_{C_{0}\left(\mathbb{R}^{d}\right)}<k^{-1}\right\} .
$$

By the $\lambda$ measurability of $\phi$ we have that $\left\|\phi(t)-g_{n}\right\|_{C_{0}\left(\mathbb{R}^{d}\right)}$ as a function of $t$ is measurable, thus $E_{k, n}$ is Borel measurable. By the density of the sequence $g_{n}$ in $C_{0}\left(\mathbb{R}^{d}\right)$ we have $(0, T)=\cup_{n \in \mathbb{N}} E_{k, n}$. Let us define $G_{k, 1}=E_{k, 1}$ and for $n \in \mathbb{N} \backslash\{1\}, G_{k, n}=E_{k, n} \backslash\left(\cup_{i=1}^{n-1} E_{k, i}\right)$. Then for $n \in \mathbb{N}, G_{k, n}$ are disjoint and cover $(0, T)$. Let us define the simple function $p_{k, n}=\sum_{i=1}^{n} 1_{G_{k, i}} g_{i}$. We have the estimate

$$
\left\|\phi-p_{k, n}\right\|_{L^{1}\left(0, T, C_{0}\left(\mathbb{R}^{d}\right)\right)} \leq \frac{T}{k}+\int_{(0, T) \backslash \cup_{i=1}^{n} G_{k, i}}\|\phi(t)\|_{C_{0}\left(\mathbb{R}^{d}\right)} d t .
$$

Let $A_{k, n}=(0, T) \backslash \cup_{i=1}^{n} G_{k, i}$ then $A_{k, n+1} \subset A_{k, n}$ and $\emptyset=\cap_{n \in \mathbb{N}} A_{k, n}$. Because $\|\phi(t)\|_{C_{0}\left(\mathbb{R}^{d}\right)} \in$ $L^{1}(0, T)$ by the absolute continuity of Lebesgue integral we have $\lim _{n \rightarrow \infty} \int_{A_{k, n}}\|\phi(t)\|_{C_{0}\left(\mathbb{R}^{d}\right)} d t=0$. From this convergence and (4.23), taking for each $k$ the $n=n_{k}$ sufficiently large we obtain $p_{k, n_{k}} \rightarrow \phi$ in $L^{1}\left(0, T, C_{0}\left(\mathbb{R}^{d}\right)\right)$.

Step 7. In this step our aim is to prove (4.10). Let $\phi \in L^{1}\left(0, T, C_{0}\left(\mathbb{R}^{d}\right)\right)$ then by the previous step there exists a sequence $\phi_{q} \in L^{1}\left(0, T, H^{m}\left(\mathbb{R}^{d}\right)\right)$ such that $\phi_{q} \rightarrow \phi$ in $L^{1}\left(0, T, C_{0}\left(\mathbb{R}^{d}\right)\right)$. By (4.9) we have

$$
\left|\int_{0}^{T} \int_{\mathbb{R}^{d}} \phi \nu_{k_{n_{\ell}}}(t)(d x) d t-\int_{0}^{T} \int_{\mathbb{R}^{d}} \phi_{q} \nu_{k_{n_{\ell}}}(t)(d x) d t\right| \leq \Lambda\left\|\phi-\phi_{q}\right\|_{L^{1}\left(0, T, C_{0}\left(\mathbb{R}^{d}\right)\right)}
$$

from which it follows that the left hand side converges to 0 as $q \rightarrow \infty$ uniformly with respect to $\ell$. We write

$$
\begin{aligned}
\int_{0}^{T} & \int_{\mathbb{R}^{d}} \phi(t)(x) \nu_{k_{n_{\ell}}}(t)(d x) d t-\int_{0}^{T} \int_{\mathbb{R}^{d}} \phi(t)(x) \nu(t)(d x) d t \\
= & \int_{0}^{T} \int_{\mathbb{R}^{d}} \phi \nu_{k_{n_{\ell}}}(t)(d x) d t-\int_{0}^{T} \int_{\mathbb{R}^{d}} \phi_{q} \nu_{k_{n_{\ell}}}(t)(d x) d t \\
& +\int_{0}^{T} \int_{\mathbb{R}^{d}} \phi_{q} \nu_{k_{n_{\ell}}}(t)(d x) d t-\int_{0}^{T} \int_{\mathbb{R}^{d}} \phi_{q} \nu(t)(d x) d t \\
& +\int_{0}^{T} \int_{\mathbb{R}^{d}} \phi_{q} \nu(t)(d x) d t-\int_{0}^{T} \int_{\mathbb{R}^{d}} \phi \nu(t)(d x) d t
\end{aligned}
$$

using the uniform convergence described above first by choosing $q$ large enough we can make the first and third terms on the right hand side small then fixing $q$ and choosing $\ell$ large enough using (4.18) we make the second term small. 
Lemma 4.4. Let there exists $\Lambda>0$ and for each $r \in \mathbb{N}, \Lambda_{r}>0$ such that

$$
\left\|\nu_{k}\right\|_{L_{w^{*}}^{\infty}\left(0, T, \mathcal{M}\left(\overline{B_{r}}\right)\right)} \leq \frac{\Lambda_{r}}{k}+\Lambda, \quad \forall k \in \mathbb{N}
$$

then there exists a subsequence $k_{n}$ and $\nu \in L_{w^{*}}^{\infty}\left(0, T, \mathcal{M}\left(\mathbb{R}^{d}\right)\right)$ such that for all compact $K \subset \mathbb{R}^{d}$

$$
\int_{0}^{T} \int_{\mathbb{R}^{d}} \phi \nu_{k_{n}}(t)(d x) d t \rightarrow \int_{0}^{T} \int_{\mathbb{R}^{d}} \phi(t, x) \nu(t)(d x) d t, \quad \forall \phi \in L^{1}\left(0, T, C_{c}(K)\right)
$$

and $\|\nu\|_{L_{w^{*}}^{\infty}\left(0, T, \mathcal{M}\left(\mathbb{R}^{d}\right)\right)} \leq \Lambda$.

If for an open set $U \subset \mathbb{R}^{d}$ and each $k \in \mathbb{N}$ we have $\nu_{k}(t)(U)=0$ for a.e. $t \in(0, T)$ then $\nu(t)(U)=0$ for a.e. $t \in(0, T)$.

Proof. Step 1. Defining $\beta_{k}$.

Let for $k \in \mathbb{N}, r_{k} \in \mathbb{N}$ be a non-decreasing sequence such that $r_{k} \rightarrow \infty$ and

$$
\frac{\Lambda_{r_{k}}}{k} \leq \Lambda_{1} \quad \text { and } \quad \frac{\Lambda_{r_{k}}}{k} \rightarrow 0 \quad \text { as } k \rightarrow \infty .
$$

Let us define $\beta_{k}:(0, T) \rightarrow \mathcal{M}\left(\mathbb{R}^{d}\right)$ by

$$
\beta_{k}=\left.\nu_{k}\right|_{\overline{B_{k}}}
$$

Step 2. $\beta_{k} \in L_{w^{*}}^{\infty}\left(0, T, \mathcal{M}\left(\mathbb{R}^{d}\right)\right)$ and uniformly bounded.

Let us show that $\beta_{k}$ is $w^{*} \lambda$-measurable. Let $\phi \in C_{0}\left(\mathbb{R}^{d}\right)$ then $\phi \in C\left(\overline{B_{r_{k}}}\right)$ and because $\nu_{k} \in$ $L_{w^{*}}^{\infty}\left(0, T, \mathcal{M}\left(\overline{B_{r_{k}}}\right)\right)$ we have

$$
\left\langle\beta_{k}(t), \phi\right\rangle=\left\langle\left.\nu_{k}(t)\right|_{\overline{B_{r_{k}}}}, \phi(\cdot)\right\rangle
$$

is $\lambda$-measurable. Thus $\beta_{k}$ is $w^{*} \lambda$-measurable. Now from the separability of $C_{0}\left(\mathbb{R}^{d}\right)$ it follows that $\left\|\beta_{k}(t)\right\|_{\mathcal{M}\left(\mathbb{R}^{d}\right)}$ is measurable.

Because of the bound (4.24) we have

$$
\left\|\beta_{k}\right\|_{L_{w^{*}}^{\infty}\left(0, T, \mathcal{M}\left(\mathbb{R}^{d}\right)\right)} \leq \frac{\Lambda_{r_{k}}}{k}+\Lambda \leq \Lambda_{1}+\Lambda
$$

Step 3. Apply the previous lemma to the sequence $\beta_{k}$.

By the uniform bound (4.26) and the previous lemma there exists a subsequence $k_{n}$ and $\nu \in L_{w^{*}}^{\infty}(0, T$, $\mathcal{M}\left(\mathbb{R}^{d}\right)$ ) such that

$$
\int_{0}^{T} \int_{\mathbb{R}^{d}} \phi \beta_{k_{n}}(t)(d x) d t \rightarrow \int_{0}^{T} \int_{\mathbb{R}^{d}} \phi \nu(t)(d x) d t, \quad \forall \phi \in L^{1}\left(0, T, C_{0}\left(\mathbb{R}^{d}\right)\right)
$$

and

$$
\|\nu\|_{L_{w^{*}}^{\infty}\left(0, T, \mathcal{M}\left(\mathbb{R}^{d}\right)\right)} \leq \liminf _{k \rightarrow \infty}\left\|\beta_{k}\right\|_{L_{w^{*}}^{\infty}\left(0, T, \mathcal{M}\left(\mathbb{R}^{d}\right)\right)} \leq \Lambda
$$

Step 4. Proving (4.25).

Let $K \subset \mathbb{R}^{d}$ be a compact set and $\phi \in L^{1}\left(0, T, C_{c}(K)\right)$ then for $n$ large enough such that $K \subset \overline{B_{r_{k_{n}}}}$ by (4.27) we have

$$
\int_{0}^{T} \int_{\mathbb{R}^{d}} \phi \nu_{k_{n}}(t)(d x) d t=\int_{0}^{T} \int_{\mathbb{R}^{d}} \phi \beta_{k_{n}}(t)(d x) d t \rightarrow \int_{0}^{T} \int_{\mathbb{R}^{d}} \phi \nu(t)(d x) d t .
$$

Step 5. Proof of the last claim.

Let $K$ a compact subset of $U$. For $\phi \in L^{1}\left(0, T, C_{c}(K)\right)$ we have for each $k \in \mathbb{N}, \int_{0}^{T} \int_{\mathbb{R}^{d}} \phi \nu_{k_{n}}(t)(d x) d t=0$ hence by (4.25) we have $\int_{0}^{T} \int_{\mathbb{R}^{d}} \phi \nu(t)(d x) d t=0$. 
Thus for all compact subsets $K \subset U$ we have

$$
\int_{0}^{T} \int_{\mathbb{R}^{d}} \phi \nu(t)(d x) d t=0 \quad \text { for all } \phi \in L^{1}\left(0, T, C_{c}(K)\right) .
$$

Let the sequence $\psi_{n} \in C_{c}(U)$ be dense in $C_{0}(\bar{U})$. Choosing $\phi=1_{I}(t) \psi_{n}(x)$ for some interval $I \subset(0, T)$ we have $\int_{I} \int_{\mathbb{R}^{d}} \psi_{n} \nu(t)(d x) d t=0$. Now because this holds for all intervals $I \subset(0, T)$ we obtain that there exists $E_{n} \in \mathcal{B}((0, T))$ with $\lambda\left(E_{n}\right)=0$ such that

$$
\int_{\mathbb{R}^{d}} \psi_{n}(x) \nu(t)(d x)=0 \quad \text { for } t \in(0, T) \backslash E_{n} .
$$

Let $E=\cup_{n \in \mathbb{N}} E_{n}$ then $\lambda(E)=0$ and

$$
\int_{\mathbb{R}^{d}} \psi_{n}(x) \nu(t)(d x)=0 \quad \text { for } t \in(0, T) \backslash E \text { and } n \in \mathbb{N}
$$

hence for $t \in(0, T) \backslash E$ we have $\nu(t)(U)=0$.

\subsection{Existence}

In the proof of Theorem 1.1 we will need the compactness result of Aubin. For ease of reading we bring here the statement of this result as it is in [10] for the special cases that we will need.

Theorem 4.5 (Aubin's compactness result). Let $X_{1}, X_{2}$ and $X_{3}$ be normed linear spaces and $T>0$. Let $f: X_{1} \rightarrow X_{2}$ be linear and compact and $g: X_{2} \rightarrow X_{3}$ be linear, bounded and injective. If for $n \in \mathbb{N}$, $v_{n} \in L^{2}\left(0, T, X_{1}\right)$ and $v \in L^{2}\left(0, T, X_{1}\right)$ such that $v_{n} \rightarrow v$ weakly in $L^{2}\left(0, T, X_{1}\right)$ and

$$
\frac{d}{d t} g\left(f\left(v_{n}\right)\right) \text { uniformly bounded in } L^{1}\left(0, T, X_{3}\right)
$$

then we have $f\left(v_{n}\right) \rightarrow f(v)$ in $L^{2}\left(0, T, X_{2}\right)$.

Proof of Theorem 1.1. Step 1. Passing to the limit in the transport equation and obtaining (1.16).

By (3.14) and (3.8) we have

$$
\left\|\chi_{\epsilon}\right\|_{L^{2}\left(0, T, L^{2}\left(\mathbb{R}^{3}\right)\right)} \leq C\left\|\chi_{0}\right\|_{L^{2}\left(\mathbb{R}^{3}\right)} .
$$

Now by (4.28) and Lemma 3.11 there exists a sequence $\epsilon_{k}$ and

$$
\chi \in L^{2}\left(0, T, L^{2}\left(\mathbb{R}^{3}\right)\right), u \in L^{\infty}\left(0, T, E^{0}\right) \cap L^{2}\left(0, T, E^{1}\right)
$$

such that

$$
\begin{aligned}
\|u\|_{L^{\infty}\left(0, T, E^{0}\right)}^{2}+\|u\|_{L^{2}\left(0, T, E^{1}\right)}^{2} & \leq C\left(\left\|\chi_{0}\right\|_{B V\left(\mathbb{R}^{3}\right)}+\left\|u_{0}\right\|_{E^{0}}^{2}\right), \\
\chi_{\epsilon_{k}} & \rightarrow \chi \text { weakly in } L^{2}\left(0, T, L^{2}\left(\mathbb{R}^{3}\right)\right)
\end{aligned}
$$

and

$$
u_{\epsilon_{k}} \rightarrow u \text { weakly in } L^{2}\left(0, T, E^{1}\right) .
$$

Let $\zeta \in C^{1}\left(\mathbb{R}^{3}\right), \zeta>0$ and $\zeta(x) \rightarrow 0$ as $|x| \rightarrow \infty$.

Let $f: L^{2}\left(\mathbb{R}^{3} ; \zeta\right) \rightarrow H^{-1}\left(\mathbb{R}^{3}\right)$ be defined for $\chi \in L^{2}\left(\mathbb{R}^{3} ; \zeta\right)$ by $\langle f(\chi), \varphi\rangle_{H^{-1}\left(\mathbb{R}^{3}\right), H^{1}\left(\mathbb{R}^{3}\right)}=(\chi, \varphi)_{L^{2}\left(\mathbb{R}^{3} ; \zeta\right)}$ for $\varphi \in H^{1}\left(\mathbb{R}^{3}\right)$.

We claim that

$$
f\left(\chi_{\epsilon_{k}}\right) \rightarrow f(\chi) \text { in } L^{2}\left(0, T, H^{-1}\left(\mathbb{R}^{3}\right)\right) .
$$

To apply Aubin's theorem let us choose $X_{1}=L^{2}\left(\mathbb{R}^{3} ; \zeta\right), X_{2}=X_{3}=H^{-1}\left(\mathbb{R}^{3}\right)$.

It is easy to see that

$$
f=f_{1}^{*} \circ f_{2}^{-1}
$$


where $f_{2}:\left(L^{2}\left(\mathbb{R}^{3} ; \zeta\right)\right)^{*} \rightarrow L^{2}\left(\mathbb{R}^{3} ; \zeta\right)$ is the Riesz representation function which has a continuous inverse and $f_{1}: H^{1}\left(\mathbb{R}^{3}\right) \rightarrow L^{2}\left(\mathbb{R}^{3} ; \zeta\right)$ is the natural embedding function. It is easy to see that $H^{1}\left(\mathbb{R}^{3}\right)$ is compactly embedded in $L^{2}\left(\mathbb{R}^{3} ; \zeta\right)$, i.e. $f_{1}$ is compact and thus $f_{1}^{*}$ is compact. Therefore $f$ is a compact operator.

Also because $X_{2}=X_{3}, X_{2}$ is injectively embedded by the identity map in $X_{3}$.

By (4.28), $\chi_{\epsilon_{k}}$ is uniformly bounded in $L^{2}\left(0, T, L^{2}\left(\mathbb{R}^{3} ; \zeta\right)\right)$ and by Lemma $4.1, \partial_{t} \chi_{\epsilon}$ is uniformly bounded in $L^{2}\left(0, T, H^{-1}\left(\mathbb{R}^{3}\right)\right)$ and therefore in $L^{1}\left(0, T, H^{-1}\left(\mathbb{R}^{3}\right)\right)$. Thus by the Aubin theorem there exists a subsequence of $\epsilon_{k}$ which we again denote by $\epsilon_{k}$ and $\gamma \in L^{2}\left(0, T, H^{-1}\left(\mathbb{R}^{3}\right)\right)$ such that

$$
f\left(\chi_{\epsilon_{k}}\right) \rightarrow \gamma \quad \text { in } L^{2}\left(0, T, H^{-1}\left(\mathbb{R}^{3}\right)\right) .
$$

Now we have for $\varphi \in L^{2}\left(0, T, H^{1}\left(\mathbb{R}^{3}\right)\right)$

$$
\begin{aligned}
\langle\gamma, \varphi\rangle & =\lim _{k \rightarrow \infty} \int_{0}^{T}\left\langle f\left(\chi_{\epsilon_{k}}\right), \varphi(t)\right\rangle_{\left(H^{1}\left(\mathbb{R}^{3}\right)\right)^{*}, H^{1}\left(\mathbb{R}^{3}\right)} d t \\
& =\lim _{k \rightarrow \infty} \int_{0}^{T} \int_{\mathbb{R}^{3}} \chi_{\epsilon_{k}} \varphi(t) \zeta d x d t \\
& =\int_{0}^{T} \int_{\mathbb{R}^{3}} \chi \varphi(t) \zeta d x d t=\int_{0}^{T}\langle f(\chi(t)), \varphi(t)\rangle_{H^{-1}\left(\mathbb{R}^{3}\right), H^{1}\left(\mathbb{R}^{3}\right)} d t
\end{aligned}
$$

hence $\gamma=f(\chi)$ and (4.32) follows from (4.33).

We claim that

$$
\Psi_{\epsilon_{k}}\left(u_{\epsilon_{k}}\right) \rightarrow u \quad \text { weakly in } L^{2}\left(0, T,\left(H^{1}\left(\mathbb{R}^{3}\right)\right)^{3}\right) .
$$

To prove (4.34) let $v \in L^{2}\left(0, T,\left(H^{1}\left(\mathbb{R}^{3}\right)\right)^{3}\right)$ then

$$
\left(v, \Psi_{\epsilon_{k}}\left(u_{\epsilon_{k}}\right)\right)_{L^{2}\left(0, T,\left(H^{1}\left(\mathbb{R}^{3}\right)\right)^{3}\right)}=\left(\Psi_{\epsilon_{k}}^{*}(v), u_{\epsilon_{k}}\right)_{L^{2}\left(0, T,\left(H^{1}\left(\mathbb{R}^{3}\right)\right)^{3}\right)}
$$

now as mentioned in Lemma 3.4 because $\Psi_{\epsilon_{k}}^{*}(v)$ converges strongly in $\left(L^{2}\left(0, T,\left(H^{1}\left(\mathbb{R}^{3}\right)\right)^{3}\right)\right)^{*}$ to $P^{*} v$ we obtain

$$
\begin{aligned}
\left(\Psi_{\epsilon_{k}}^{*}(v), u_{\epsilon_{k}}\right)_{L^{2}\left(0, T,\left(H^{1}\left(\mathbb{R}^{3}\right)\right)^{3}\right)} & \rightarrow\left(P^{*} v, u\right)_{L^{2}\left(0, T,\left(H^{1}\left(\mathbb{R}^{3}\right)\right)^{3}\right)} \\
& =(v, P u)_{L^{2}\left(0, T,\left(H^{1}\left(\mathbb{R}^{3}\right)\right)^{3}\right)}=(v, u)_{L^{2}\left(0, T,\left(H^{1}\left(\mathbb{R}^{3}\right)\right)^{3}\right)}
\end{aligned}
$$

which proves (4.34).

By (3.12) for $\varphi \in C_{c}^{\infty}\left((-\infty, T) \times \mathbb{R}^{3}\right)$ we have

$$
\begin{aligned}
& -\int_{\mathbb{R}^{3}} \chi_{0, \epsilon} \varphi(0, x) d x-\int_{0}^{\infty} \int_{\mathbb{R}^{3}} \chi_{\epsilon} \partial_{t} \varphi d x d t \\
& -\int_{0}^{\infty} \int_{\mathbb{R}^{3}} \chi_{\epsilon} \Psi_{\epsilon}\left(u_{\epsilon}\right) \cdot \nabla \varphi d x d t+\epsilon \int_{0}^{\infty} \int_{\mathbb{R}^{3}} \nabla \chi_{\epsilon} \cdot \nabla \varphi d x d t=0 .
\end{aligned}
$$

Our aim is now for the sequence $\epsilon_{k}$ to pass to the limit in (4.35). We pass to the limit in the first term in (4.35) using the convergence $\chi_{0, \epsilon_{k}} \rightarrow \chi_{0}$ in $L^{2}\left(\mathbb{R}^{3}\right)$. In the second term in (4.35) we pass to the limit using the weak convergence (4.30). To pass to the limit in the third term in (4.35), by (4.32) and (4.34) we have

$$
\begin{aligned}
\int_{0}^{T} \int_{\mathbb{R}^{3}} \chi_{\epsilon_{k}} \Psi_{\epsilon_{k}}\left(u_{\epsilon_{k}}\right) \cdot \nabla \varphi d x d t & =\int_{0}^{T} \int_{\mathbb{R}^{3}} \chi_{\epsilon_{k}} \Psi_{\epsilon_{k}}\left(u_{\epsilon_{k}}\right) \cdot\left(\zeta^{-1} \nabla \varphi\right) \zeta d x d t \\
& =\int_{0}^{T}\left\langle f\left(\chi_{\epsilon_{k}}\right), \Psi_{\epsilon_{k}}\left(u_{\epsilon_{k}}\right) \cdot\left(\zeta^{-1} \nabla \varphi\right)\right\rangle_{H^{-1}\left(\mathbb{R}^{3}\right), H^{1}\left(\mathbb{R}^{3}\right)} d t \\
& \rightarrow \int_{0}^{T}\left\langle f(\chi), u \cdot\left(\zeta^{-1} \nabla \varphi\right)\right\rangle_{H^{1-}\left(\mathbb{R}^{3}\right), H^{1}\left(\mathbb{R}^{3}\right)} d t \\
& =\int_{0}^{T} \int_{\mathbb{R}^{3}} \chi u \cdot\left(\zeta^{-1} \nabla \varphi\right) \zeta d x d t=\int_{0}^{T} \int_{\mathbb{R}^{3}} \chi u \cdot \nabla \varphi d x d t .
\end{aligned}
$$


For the fourth term in (4.35) by (3.14) and (3.8) we have

$$
\begin{aligned}
& \left|\epsilon \int_{0}^{T} \int_{\mathbb{R}^{3}} \nabla \chi_{\epsilon} \cdot \nabla \varphi d x d t\right| \\
& \quad \leq \sqrt{\epsilon}\left\{\epsilon \int_{0}^{T} \int_{\mathbb{R}^{3}}\left|\nabla \chi_{\epsilon}\right|^{2} d x d t\right\}^{\frac{1}{2}}\left\{\int_{0}^{T} \int_{\mathbb{R}^{3}}|\nabla \varphi|^{2} d x d t\right\}^{\frac{1}{2}} \\
& \quad \leq C \sqrt{\epsilon}\left\|\chi_{0, \epsilon}\right\|_{L^{2}\left(\mathbb{R}^{3}\right)}\left\{\int_{0}^{T} \int_{\mathbb{R}^{3}}|\nabla \varphi|^{2} d x d t\right\}^{\frac{1}{2}} \\
& \quad \leq C \sqrt{\epsilon}\left\|\chi_{0}\right\|_{L^{2}\left(\mathbb{R}^{3}\right)}\left\{\int_{0}^{T} \int_{\mathbb{R}^{3}}|\nabla \varphi|^{2} d x d t\right\}^{\frac{1}{2}} \rightarrow 0 .
\end{aligned}
$$

Thus by passing to the limit for $\epsilon=\epsilon_{k}$ in (4.35) we obtain for all $\varphi \in C_{c}^{\infty}\left((-\infty, T) \times \mathbb{R}^{3}\right)$

$$
-\int_{\mathbb{R}^{3}} \chi_{0} \varphi(0, x) d x-\int_{0}^{T} \int_{\mathbb{R}^{3}} \chi \partial_{t} \varphi d x d t-\int_{0}^{T} \int_{\mathbb{R}^{3}} \chi u \cdot \nabla \varphi d x d t=0
$$

thus $\chi$ is the renormalized solution of (1.16).

Step 2. Obtaining the terms on the left hand side of (1.17).

Because $u_{\epsilon}$ satisfies (3.11) we have for all $\varphi \in\left(C_{c}^{\infty}\left((-\infty, T) \times \mathbb{R}^{3}\right)\right)^{3}$ with $\operatorname{div}(\varphi)=0$

$$
\begin{aligned}
& -\int_{\mathbb{R}^{3}} u_{0}^{T} \varphi(0) d x-\int_{0}^{T} \int_{\mathbb{R}^{3}} u_{\epsilon}^{T} \partial_{t} \varphi d x d t \\
& \quad-\int_{0}^{T} \int_{\mathbb{R}^{3}}\left(\Psi_{\epsilon}\left(u_{\epsilon}\right) \otimes u_{\epsilon}\right): \nabla \varphi d x d t+\int_{0}^{T} \int_{\mathbb{R}^{3}} D u_{\epsilon}: D \varphi d x d t \\
& =\int_{0}^{T} \int_{\mathbb{R}^{3}} \frac{\nabla \chi_{\epsilon} \otimes \nabla \chi_{\epsilon}}{\left(\left|\nabla \chi_{\epsilon}\right|^{2}+\epsilon^{2}\right)^{\frac{1}{2}}}: \nabla \Psi_{\epsilon}(\varphi) d x d t .
\end{aligned}
$$

For the second term on the left hand side of (4.36) by the weak convergence (4.31) we have

$$
\int_{0}^{T} \int_{\mathbb{R}^{3}} u_{\epsilon_{k}}^{T} \partial_{t} \varphi d x d t \rightarrow \int_{0}^{T} \int_{\mathbb{R}^{3}} u^{T} \partial_{t} \varphi d x d t .
$$

To pass to the limit in the third term on the left hand side of (4.36) let us notice that by Lemma 3.11 we have $\sup _{0<\epsilon<1}\left\|u_{\epsilon}\right\|_{L^{2}\left(0, T, E^{1}\right)}<\infty$ and also by Lemma 4.2 we have $\sup _{0<\epsilon<1}\left\|\partial_{t} u_{\epsilon}\right\|_{L^{1}\left(0, T,\left(E^{3}\right)^{*}\right)}<\infty$.

Let $\omega$ be as in Proposition 3.3. Let us define

$$
E_{\omega}^{0}=\text { closure of }\left\{v \in\left(C_{c}^{\infty}\left(\mathbb{R}^{3}\right)\right)^{3} \mid \operatorname{div}(v)=0\right\} \quad \text { in }\left(L^{2}\left(\mathbb{R}^{3} ; \omega\right)\right)^{3} .
$$

To apply the Aubin theorem let us choose $X_{1}=E^{1}, X_{2}=E_{\omega}^{0}$ and $X_{3}=\left(E^{3}\right)^{*}$.

It is easy to check that the natural embedding of $E^{1}$ in $E_{\omega}^{0}$ is compact, let $f$ be this compact embedding.

We have that $E^{3}$ is densely and continuously embedded in $E^{0}$ and in turn this is densely and continuously embedded in $E_{\omega}^{0}$. Finally $E_{\omega}^{0}$ as a Hilbert space is isometrically isomorphic to its dual $\left(E_{\omega}^{0}\right)^{*}$. Hence $E^{3}$ is densely and continuously embedded in $\left(E_{\omega}^{0}\right)^{*}$. Now it follows that $E_{\omega}^{0}$ is injectively and continuously embedded in $\left(E^{3}\right)^{*}$ and let $g$ be this embedding.

Hence we may apply the theorem of Aubin to obtain that for a subsequence that we denote again by $\epsilon_{k}, u_{\epsilon_{k}} \rightarrow u$ in $L^{2}\left(0, T, E_{\omega}^{0}\right)$.

By Lemma 3.4 we compute

$$
\begin{aligned}
\left\|\Psi_{\epsilon_{k}}\left(u_{\epsilon_{k}}\right)-u\right\|_{L^{2}\left(0, T,\left(L^{2}\left(\mathbb{R}^{3} ; \omega\right)\right)^{3}\right)} & \leq\left\|\Psi_{\epsilon_{k}}\left(u_{\epsilon_{k}}-u\right)\right\|_{L^{2}\left(0, T,\left(L^{2}\left(\mathbb{R}^{3} ; \omega\right)\right)^{3}\right)}+\left\|\Psi_{\epsilon_{k}}(u)-u\right\|_{L^{2}\left(0, T,\left(L^{2}\left(\mathbb{R}^{3} ; \omega\right)\right)^{3}\right)} \\
& \leq C\left\|u_{\epsilon_{k}}-u\right\|_{L^{2}\left(0, T,\left(L^{2}\left(\mathbb{R}^{3} ; \omega\right)\right)^{3}\right)}+\left\|\Psi_{\epsilon_{k}}(u)-u\right\|_{L^{2}\left(0, T,\left(L^{2}\left(\mathbb{R}^{3} ; \omega\right)\right)^{3}\right)}
\end{aligned}
$$


thus

$$
\Psi_{\epsilon_{k}}\left(u_{\epsilon_{k}}\right) \rightarrow u \quad \text { in } L^{2}\left(0, T, E_{\omega}^{0}\right) .
$$

Finally using the fact that $\varphi$ has compact support from (4.31) and (4.37) we obtain

$$
-\int_{0}^{T} \int_{\mathbb{R}^{3}}\left(\Psi_{\epsilon_{k}}\left(u_{\epsilon_{k}}\right) \otimes u_{\epsilon_{k}}\right): \nabla \varphi d x d t \rightarrow-\int_{0}^{T} \int_{\mathbb{R}^{3}}(u \otimes u): \nabla \varphi d x d t .
$$

For the fourth term on the left hand side of (4.36) by (4.31) we have

$$
\int_{0}^{T} \int_{\mathbb{R}^{3}} D u_{\epsilon_{k}}: D \varphi d x d t \rightarrow \int_{0}^{T} \int_{\mathbb{R}^{3}} D u: D \varphi d x d t .
$$

Step 3. Existence of the varifold and obtaining the term on the right hand side of (1.17).

Let $r \in \mathbb{N}$. We compute for $0<t<T$

$$
\begin{aligned}
\int_{B_{r}}\left|\nabla \chi_{\epsilon}\right| d x & \leq \int_{B_{r}}\left(\left|\nabla \chi_{\epsilon}\right|^{2}+\epsilon^{2}\right)^{\frac{1}{2}} d x \leq \epsilon\left|B_{r}\right|+\int_{B_{r}}\left\{\left(\left|\nabla \chi_{\epsilon}\right|^{2}+\epsilon^{2}\right)^{\frac{1}{2}}-\epsilon\right\} d x \\
& \leq \epsilon\left|B_{r}\right|+\int_{\mathbb{R}^{3}}\left\{\left(\left|\nabla \chi_{\epsilon}\right|^{2}+\epsilon^{2}\right)^{\frac{1}{2}}-\epsilon\right\} d x
\end{aligned}
$$

hence by Lemma 3.11 there exists $\Lambda>0$ such that

$$
\left\|\nabla \chi_{\epsilon}\right\|_{L^{\infty}\left(0, T,\left(L^{1}\left(B_{r}\right)\right)^{3}\right)} \leq \epsilon\left|B_{r}\right|+\Lambda .
$$

By the embedding $L^{1}\left(B_{r}\right) \hookrightarrow \mathcal{M}\left(\overline{B_{r}}\right)$ we have

$$
\left\|\nabla \chi_{\epsilon}\right\|_{L^{\infty}\left(0, T,\left(\mathcal{M}\left(\overline{B_{r}}\right)\right)^{3}\right)} \leq \epsilon\left|B_{r}\right|+\Lambda \text {. }
$$

Then by Lemma 4.4 there exists a subsequence of $\epsilon_{k}$ which we again denote by $\epsilon_{k}$ and $\mu \in L_{w^{*}}^{\infty}(0, T$, $\left.\left(\mathcal{M}\left(\mathbb{R}^{3}\right)\right)^{3}\right)$ such that

$$
\int_{0}^{T} \int_{\mathbb{R}^{3}} \varphi \cdot \nabla \chi_{\epsilon_{k}} d x d t \rightarrow \int_{0}^{T} \int_{\mathbb{R}^{3}} \varphi \cdot \mu(t)(d x) d t, \quad \forall \varphi \in\left(C_{c}\left(\mathbb{R}^{4}\right)\right)^{3}
$$

and

$$
\|\mu\|_{L_{w^{*}}^{\infty}\left(0, T,\left(\mathcal{M}\left(\mathbb{R}^{3}\right)\right)^{3}\right)} \leq C \Lambda .
$$

Also for $\varphi \in\left(C_{c}^{1}\left(\mathbb{R}^{4}\right)\right)^{3}$

$$
\int_{0}^{T} \int_{\mathbb{R}^{3}} \varphi \cdot \nabla \chi_{\epsilon_{k}} d x d t=-\int_{0}^{T} \int_{\mathbb{R}^{3}} \operatorname{div}(\varphi) \chi_{\epsilon_{k}} d x d t \rightarrow-\int_{0}^{T} \int_{\mathbb{R}^{3}} \operatorname{div}(\varphi) \chi d x d t
$$

as $k \rightarrow \infty$.

Thus by (4.40) and (4.42) we have for $\varphi \in\left(C_{c}^{1}\left(\mathbb{R}^{4}\right)\right)^{3}$

$$
\int_{0}^{T} \int_{\mathbb{R}^{3}} \varphi \cdot \mu(t)(d x) d t=-\int_{0}^{T} \int_{\mathbb{R}^{3}} \operatorname{div}(\varphi) \chi d x d t
$$

hence as distributions

$$
\mu(t)=\nabla \chi(t, \cdot) \quad \text { for a.e. } 0<t<T .
$$

Let us define for $0<t<T$ and $0<\epsilon<1$ the linear functional $V_{\epsilon}(t)$ on $C_{c}\left(\mathbb{R}^{6}\right)$ by

$$
\left\langle V_{\epsilon}(t), \varphi\right\rangle=\int_{\mathbb{R}^{3} \cap\left\{\nabla \chi_{\epsilon} \neq 0\right\}} \varphi\left(x,-\frac{\nabla \chi_{\epsilon}}{\left|\nabla \chi_{\epsilon}\right|}\right)\left|\nabla \chi_{\epsilon}\right| d x \quad \text { for } \varphi \in C_{c}\left(\mathbb{R}^{6}\right) .
$$

As a positive linear functional on $C_{c}\left(\mathbb{R}^{6}\right)$ by Riesz representation theorem $V_{\epsilon}(t)$ corresponds to a unique Radon measure on $\mathbb{R}^{6}$ which we again denote by $V_{\epsilon}(t)$.

For a.e. $0<t<T$ and $r \in \mathbb{N}$ we have by (4.38)

$$
\left\|V_{\epsilon}(t)\right\|_{\mathcal{M}\left(\overline{B_{r}^{6}}\right)} \leq\left\|V_{\epsilon}(t)\right\|_{\mathcal{M}\left(\overline{B_{r}^{3}} \times \mathbb{R}^{3}\right)} \leq\left\|\nabla \chi_{\epsilon}(t)\right\|_{L^{1}\left(B_{r}^{3}\right)} \leq \epsilon\left|B_{r}^{3}\right|+\Lambda .
$$


Now let us show that $V_{\epsilon}(t)$ with values in $\mathcal{M}\left(\overline{B_{r}^{6}}\right)$ is $w^{*} \lambda$-measureable. Let $\varphi \in C\left(\overline{B_{r}^{6}}\right)$ then by the dominated convergence theorem we have

$$
\begin{aligned}
\left\langle V_{\epsilon}(t), \varphi\right\rangle_{\mathcal{M}\left(\overline{B_{r}^{6}}\right), C\left(\overline{B_{r}^{6}}\right)} & =\int_{\mathbb{R}^{3} \cap\left\{\nabla \chi_{\epsilon} \neq 0\right\}} \varphi\left(x,-\frac{\nabla \chi_{\epsilon}}{\left|\nabla \chi_{\epsilon}\right|}\right)\left|\nabla \chi_{\epsilon}\right| 1_{\left(x,-\frac{\nabla \chi_{\epsilon}}{\left|\nabla \chi_{\epsilon}\right|}\right) \in \overline{B_{r}^{6}}} d x \\
& =\lim _{\ell \rightarrow \infty} \int_{\mathbb{R}^{3}} \varphi\left(x,-\frac{\nabla \chi_{\epsilon}}{\left|\nabla \chi_{\epsilon}\right|+\frac{1}{\ell}}\right)\left|\nabla \chi_{\epsilon}\right| 1_{\left(x,-\frac{\nabla \chi_{\epsilon}}{\left|\nabla \chi_{\epsilon}\right|+\frac{1}{\ell}}\right) \in \overline{B_{r}^{6}}} d x
\end{aligned}
$$

hence the right hand side as the limit of measurable functions is measurable.

By the separability of $C\left(\overline{B_{r}^{6}}\right)$ it follows that $\left\|V_{\epsilon}(t)\right\|_{\mathcal{M}\left(\overline{B_{r}^{6}}\right)}$ is measurable.

By these measurabilities and (4.44) we obtain $V_{\epsilon} \in L_{w^{*}}^{\infty}\left(0, T, \mathcal{M}\left(\overline{B_{r}^{6}}\right)\right)$ with

$$
\left\|V_{\epsilon}\right\|_{L_{w^{*}}^{\infty}\left(0, T, \mathcal{M}\left(\overline{B_{r}^{6}}\right)\right)} \leq \epsilon\left|B_{r}^{3}\right|+\Lambda .
$$

By Lemma 4.4 there exists a subsequence of $\epsilon_{k}$ which we denote again by $\epsilon_{k}$ and $V \in L_{w^{*}}^{\infty}\left(0, T, \mathcal{M}\left(\mathbb{R}^{3} \times\right.\right.$ $\left.\mathbb{S}^{2}\right)$ ) such that

$$
\int_{0}^{T} \int_{\mathbb{R}^{3} \times \mathbb{S}^{2}} \varphi V_{\epsilon_{k}}(t)(d(x, y)) d t \rightarrow \int_{0}^{T} \int_{\mathbb{R}^{3} \times \mathbb{S}^{2}} \varphi V(t)(d(x, y)) d t, \quad \forall \varphi \in C_{c}\left(\mathbb{R}^{7}\right)
$$

and

$$
\|V\|_{L_{w^{*}}^{\infty}\left(0, T, \mathcal{M}\left(\mathbb{R}^{3} \times \mathbb{S}^{2}\right)\right)} \leq \Lambda
$$

Let us prove that

$$
-\int_{0}^{T} \int_{\mathbb{R}^{3}} \varphi \cdot \mu(t)(d x) d t=\int_{0}^{T} \int_{\mathbb{R}^{3} \times \mathbb{S}^{2}} \varphi \cdot y V(t)(d(x, y)) d t, \quad \forall \varphi \in\left(C_{c}\left(\mathbb{R}^{4}\right)\right)^{3} .
$$

Let $\varphi \in\left(C_{c}\left(\mathbb{R}^{4}\right)\right)^{3}$. For $(t, x) \in \mathbb{R}^{4}$ and $y \in \mathbb{S}^{2}$ let us define $\tilde{\varphi}(t, x, y)=\varphi(t, x) \cdot y$, then one may extend $\tilde{\varphi}$ to a function in $C_{c}\left(\mathbb{R}^{7}\right)$ and by $(4.45)$ we obtain

$$
\begin{aligned}
-\int_{0}^{T} \int_{\mathbb{R}^{3}} \varphi \cdot \mu(t)(d x) d t & =-\lim _{k \rightarrow \infty} \int_{0}^{T} \int_{\mathbb{R}^{3}} \varphi \cdot \nabla \chi_{\epsilon_{k}} d x d t \\
& =\lim _{k \rightarrow \infty} \int_{0}^{T} \int_{\mathbb{R}^{3} \cap\left\{\nabla \chi_{\epsilon_{k}} \neq 0\right\}} \tilde{\varphi}\left(t, x,-\frac{\nabla \chi_{\epsilon_{k}}}{\left|\nabla \chi_{\epsilon_{k}}\right|}\right)\left|\nabla \chi_{\epsilon_{k}}\right| d x d t \\
& =\lim _{k \rightarrow \infty} \int_{0}^{T} \int_{\mathbb{R}^{3} \times \mathbb{S}^{2}} \tilde{\varphi}(t, x, y) V_{\epsilon_{k}}(t)(d(x, y)) d t \\
& =\int_{0}^{T} \int_{\mathbb{R}^{3} \times \mathbb{S}^{2}} \tilde{\varphi}(t, x, y) V(t)(d(x, y)) d t \\
& =\int_{0}^{T} \int_{\mathbb{R}^{3} \times \mathbb{S}^{2}} \varphi \cdot y V(t)(d(x, y)) d t
\end{aligned}
$$

thus we have proved (4.47). Now (1.10) follows from (4.47).

Let us prove that

$$
\begin{aligned}
& \int_{0}^{T} \int_{\mathbb{R}^{3} \times \mathbb{S}^{2}} \varphi V(t)(d(x, y)) d t \\
& \quad=\lim _{k \rightarrow \infty} \int_{0}^{T} \int_{\mathbb{R}^{3} \times \mathbb{S}^{2}} \varphi \frac{\left|\nabla \chi_{\epsilon_{k}}\right|}{\left(\left|\nabla \chi_{\epsilon_{k}}\right|^{2}+\epsilon_{k}^{2}\right)^{\frac{1}{2}}} V_{\epsilon_{k}}(t)(d(x, y)) d t, \quad \forall \varphi \in C_{c}\left(\mathbb{R}^{7}\right) .
\end{aligned}
$$


Let $\varphi \in C_{c}\left(\mathbb{R}^{7}\right)$. We compute

$$
\begin{aligned}
& \left|\int_{0}^{T} \int_{\mathbb{R}^{3} \times \mathbb{S}^{2}} \varphi V(t)(d(x, y)) d t-\int_{0}^{T} \int_{\mathbb{R}^{3} \times \mathbb{S}^{2}} \varphi \frac{\left|\nabla \chi_{\epsilon_{k}}\right|}{\left(\left|\nabla \chi_{\epsilon_{k}}\right|^{2}+\epsilon_{k}^{2}\right)^{\frac{1}{2}}} V_{\epsilon_{k}}(t)(d(x, y)) d t\right| \\
& \leq\left|\int_{0}^{T} \int_{\mathbb{R}^{3} \times \mathbb{S}^{2}} \varphi V(t)(d(x, y)) d t-\int_{0}^{T} \int_{\mathbb{R}^{3} \times \mathbb{S}^{2}} \varphi V_{\epsilon_{k}}(t)(d(x, y)) d t\right| \\
& \quad+\int_{0}^{T} \int_{\mathbb{R}^{3} \times \mathbb{S}^{2}}|\varphi|\left(1-\frac{\left|\nabla \chi_{\epsilon_{k}}\right|}{\left(\left|\nabla \chi_{\epsilon_{k}}\right|^{2}+\epsilon_{k}^{2}\right)^{\frac{1}{2}}}\right) V_{\epsilon_{k}}(t)(d(x, y)) d t .
\end{aligned}
$$

By (4.45) the first term on the right hand side of (4.49) converges to 0.

To show that the second term on the right hand side of (4.49) also converges to 0 we estimate

$$
\begin{aligned}
\frac{\left(\left|\nabla \chi_{\epsilon_{k}}\right|^{2}+\epsilon_{k}^{2}\right)^{\frac{1}{2}}-\left|\nabla \chi_{\epsilon_{k}}\right|}{\left(\left|\nabla \chi_{\epsilon_{k}}\right|^{2}+\epsilon_{k}^{2}\right)^{\frac{1}{2}}}\left|\nabla \chi_{\epsilon_{k}}\right| & =\frac{\epsilon_{k}^{2}}{\left(\left|\nabla \chi_{\epsilon_{k}}\right|^{2}+\epsilon_{k}^{2}\right)^{\frac{1}{2}}} \frac{\left|\nabla \chi_{\epsilon_{k}}\right|}{\left(\left|\nabla \chi_{\epsilon_{k}}\right|^{2}+\epsilon_{k}^{2}\right)^{\frac{1}{2}}+\left|\nabla \chi_{\epsilon_{k}}\right|} \\
& \leq \frac{\epsilon_{k}^{2}}{\left(\left|\nabla \chi_{\epsilon_{k}}\right|^{2}+\epsilon_{k}^{2}\right)^{\frac{1}{2}}} \leq \epsilon_{k}
\end{aligned}
$$

and using the fact that $\varphi$ has compact support and is bounded we compute

$$
\begin{aligned}
& \int_{0}^{T} \int_{\mathbb{R}^{3} \times \mathbb{S}^{2}}|\varphi|\left(1-\frac{\left|\nabla \chi_{\epsilon_{k}}\right|}{\left(\left|\nabla \chi_{\epsilon_{k}}\right|^{2}+\epsilon_{k}^{2}\right)^{\frac{1}{2}}}\right) V_{\epsilon_{k}}(t)(d(x, y)) d t \\
& \quad=\int_{0}^{T} \int_{\mathbb{R}^{3} \cap\left\{\nabla \chi_{\epsilon_{k}} \neq 0\right\}}\left|\varphi\left(t, x,-\frac{\nabla \chi_{\epsilon_{k}}}{\left|\nabla \chi_{\epsilon_{k}}\right|}\right)\right|\left(1-\frac{\left|\nabla \chi_{\epsilon_{k}}\right|}{\left(\left|\nabla \chi_{\epsilon_{k}}\right|^{2}+\epsilon_{k}^{2}\right)^{\frac{1}{2}}}\right)\left|\nabla \chi_{\epsilon_{k}}\right| d x d t \\
& \quad \leq \epsilon_{k} \int_{0}^{T} \int_{\mathbb{R}^{3} \cap\left\{\nabla \chi_{\epsilon_{k}} \neq 0\right\}}\left|\varphi\left(t, x,-\frac{\nabla \chi_{\epsilon_{k}}}{\left|\nabla \chi_{\epsilon_{k}}\right|}\right)\right| d x d t \leq C \epsilon_{k} \rightarrow 0 \quad \text { as } k \rightarrow \infty .
\end{aligned}
$$

Now we proved that both terms on the right hand side of (4.49) converge to 0 hence the left hand side converges to 0 and this proves (4.48).

Let us prove that for all $\varphi \in\left(C_{c}^{\infty}\left(\mathbb{R}^{4}\right)\right)^{3}$ with $\operatorname{div}(\varphi)=0$

$$
\lim _{k \rightarrow \infty} \int_{0}^{T} \int_{\mathbb{R}^{3}} \frac{\nabla \chi_{\epsilon_{k}} \otimes \nabla \chi_{\epsilon_{k}}}{\left(\left|\nabla \chi_{\epsilon_{k}}\right|^{2}+\epsilon_{k}^{2}\right)^{\frac{1}{2}}}: \nabla \Psi_{\epsilon_{k}}(\varphi) d x d t=-\int_{0}^{T}\langle\delta V(t), \varphi(t)\rangle d t .
$$

Let $\varphi \in\left(C_{c}^{\infty}\left(\mathbb{R}^{4}\right)\right)^{3}$ with $\operatorname{div}(\varphi)=0$. We write

$$
\begin{aligned}
\int_{0}^{T} \int_{\mathbb{R}^{3}} \frac{\nabla \chi_{\epsilon_{k}} \otimes \nabla \chi_{\epsilon_{k}}}{\left(\left|\nabla \chi_{\epsilon_{k}}\right|^{2}+\epsilon^{2}\right)^{\frac{1}{2}}}: \nabla \Psi_{\epsilon_{k}}(\varphi) d x d t= & \int_{0}^{T} \int_{\mathbb{R}^{3}} \frac{\nabla \chi_{\epsilon_{k}} \otimes \nabla \chi_{\epsilon_{k}}}{\left(\left|\nabla \chi_{\epsilon_{k}}\right|^{2}+\epsilon^{2}\right)^{\frac{1}{2}}}:\left(\nabla \Psi_{\epsilon_{k}}(\varphi)-\nabla \varphi\right) d x d t \\
& +\int_{0}^{T} \int_{\mathbb{R}^{3}} \frac{\nabla \chi_{\epsilon_{k}} \otimes \nabla \chi_{\epsilon_{k}}}{\left(\left|\nabla \chi_{\epsilon_{k}}\right|^{2}+\epsilon^{2}\right)^{\frac{1}{2}}}: \nabla \varphi d x d t
\end{aligned}
$$

For the first term on the right hand side of the equation above because $\nabla \Psi_{\epsilon_{k}}(\varphi)$ converges in $\left(C\left(\mathbb{R}^{4}\right)\right)^{3 \times 3}$ to $\nabla \varphi$ we obtain that

$$
\begin{aligned}
& \left|\int_{0}^{T} \int_{\mathbb{R}^{3}} \frac{\nabla \chi_{\epsilon_{k}} \otimes \nabla \chi_{\epsilon_{k}}}{\left(\left|\nabla \chi_{\epsilon_{k}}\right|^{2}+\epsilon^{2}\right)^{\frac{1}{2}}}:\left(\nabla \Psi_{\epsilon_{k}}(\varphi)-\nabla \varphi\right) d x d t\right| \\
& \quad \leq C \int_{0}^{T} \int_{\mathbb{R}^{3}}\left(\left(\left|\nabla \chi_{\epsilon_{k}}\right|^{2}+\epsilon_{k}^{2}\right)^{\frac{1}{2}}-\epsilon_{k}\right)\left|\nabla \Psi_{\epsilon_{k}}(\varphi)-\nabla \varphi\right| d x d t
\end{aligned}
$$




$$
\begin{aligned}
& \leq C T\left\{\sup _{0<t<T} \int_{\mathbb{R}^{3}}\left(\left(\left|\nabla \chi_{\epsilon_{k}}\right|^{2}+\epsilon_{k}^{2}\right)^{\frac{1}{2}}-\epsilon_{k}\right) d x\right\}\left\|\left|\nabla \Psi_{\epsilon_{k}}(\varphi)-\nabla \varphi\right|\right\|_{C_{b}\left([0, T] \times \mathbb{R}^{3}\right)} \\
& \leq C_{1}\left\|\left|\nabla \Psi_{\epsilon_{k}}(\varphi)-\nabla \varphi\right|\right\|_{C_{b}\left([0, T] \times \mathbb{R}^{3}\right)} \rightarrow 0
\end{aligned}
$$

in the last inequality we used Lemma 3.11 .

For the second term on the right hand side of the equation above using (4.48) we have

$$
\begin{aligned}
\int_{0}^{T} & \int_{\mathbb{R}^{3}} \frac{\nabla \chi_{\epsilon_{k}} \otimes \nabla \chi_{\epsilon_{k}}}{\left(\left|\nabla \chi_{\epsilon_{k}}\right|^{2}+\epsilon_{k}^{2}\right)^{\frac{1}{2}}}: \nabla \varphi d x d t \\
= & \int_{0}^{T} \int_{\mathbb{R}^{3}}\left(-\frac{\nabla \chi_{\epsilon_{k}}}{\left|\nabla \chi_{\epsilon_{k}}\right|}\right) \otimes\left(-\frac{\nabla \chi_{\epsilon_{k}}}{\left|\nabla \chi_{\epsilon_{k}}\right|}\right): \nabla \varphi \frac{\left|\nabla \chi_{\epsilon_{k}}\right|^{2}}{\left(\left|\nabla \chi_{\epsilon_{k}}\right|^{2}+\epsilon_{k}^{2}\right)^{\frac{1}{2}}} d x d t \\
= & -\int_{0}^{T} \int_{\mathbb{R}^{3}} I-\left(-\frac{\nabla \chi_{\epsilon_{k}}}{\left|\nabla \chi_{\epsilon_{k}}\right|}\right) \otimes\left(-\frac{\nabla \chi_{\epsilon_{k}}}{\left|\nabla \chi_{\epsilon_{k}}\right|}\right): \nabla \varphi \frac{\left|\nabla \chi_{\epsilon_{k}}\right|}{\left(\left|\nabla \chi_{\epsilon_{k}}\right|^{2}+\epsilon_{k}^{2}\right)^{\frac{1}{2}}}\left|\nabla \chi_{\epsilon_{k}}\right| d x d t \\
= & -\int_{0}^{T} \int_{\mathbb{R}^{3} \times \mathbb{S}^{2}}(I-y \otimes y): \nabla \varphi \frac{\left|\nabla \chi_{\epsilon_{k}}\right|}{\left(\left|\nabla \chi_{\epsilon_{k}}\right|^{2}+\epsilon_{k}^{2}\right)^{\frac{1}{2}}} V_{\epsilon_{k}}(t)(d(x, y)) d t \\
& \rightarrow-\int_{0}^{T} \int_{\mathbb{R}^{3} \times \mathbb{S}^{2}}(I-y \otimes y): \nabla \varphi(t, x) V(t)(d(x, y)) d t \\
= & -\int_{0}^{T}\langle\delta V(t), \varphi(t)\rangle d t .
\end{aligned}
$$

Now by (4.51)-(4.53) we prove (4.50).

Step 4. The inequality (1.15).

For the first two term on the left hand side of (1.15) this inequality follows from (4.29). Because $\chi$ is the renormalized solution of $(1.16)$ and $\chi_{0} \in L^{1}\left(\mathbb{R}^{3} ;\{0,1\}\right)$ we obtain that $\chi \in L^{\infty}\left(0, T, L^{1}\left(\mathbb{R}^{3} ;\{0,1\}\right)\right)$ and the bound for the third term on the left hand side of (1.15). For the fourth term on the left hand side of (1.15) this inequality follows from (4.41) and (4.43). For the fifth term on the left hand side of (1.15) this inequality follows from (4.46).

And this completes the proof of the theorem.

Proof of Theorem 1.2. In the case of axisymmetric initial values and boundary condition by the Theorem 3.12 for each $0<\epsilon<1$ there exists $u_{\epsilon} \in L^{2}\left(0, T, E_{\text {a.s. }}^{1}\right)$ and axisymmetric $\chi_{\epsilon} \in L^{2}\left(0, T, H^{1}\left(\mathbb{R}^{3}\right)\right)$, such that together these are a solution to the system of Eqs. (3.10) and (3.11).

In the following when writing $u_{\epsilon}$ or $\chi_{\epsilon}$ we mean these axisymmetric solutions.

We proceed as in the proof of Theorem 3.4 and prove the existence of a Varifold solution triple $(u, \chi, V)$ to our equations. As described in the proof of Theorem 3.4 these are appropriate limits of the regularized solutions corresponding to a sequence $\epsilon_{k}$.

In the following using the axisymmetry of the regularized solutions we prove the axisymmetry properties of the varifold solution triple.

For $\theta \in \mathbb{R}$ by the axisymmetry of $\chi_{\epsilon_{k}}$ we have $\tau_{\theta} \chi_{\epsilon_{k}}=\chi_{\epsilon_{k}}$. Thus for $\varphi \in C_{c}\left(\mathbb{R}^{4}\right)$ by the weak convergence (4.30) we have

$$
\begin{aligned}
\int_{0}^{T} \int_{\mathbb{R}^{3}} \tau_{\theta} \chi \varphi d x d t & =\int_{0}^{T} \int_{\mathbb{R}^{3}} \chi \tau_{-\theta} \varphi d x d t=\lim _{k \rightarrow \infty} \int_{0}^{T} \int_{\mathbb{R}^{3}} \chi_{\epsilon_{k}} \tau_{-\theta} \varphi d x d t \\
& =\lim _{k \rightarrow \infty} \int_{0}^{T} \int_{\mathbb{R}^{3}} \tau_{\theta} \chi_{\epsilon_{k}} \varphi d x d t=\lim _{k \rightarrow \infty} \int_{0}^{T} \int_{\mathbb{R}^{3}} \chi_{\epsilon_{k}} \varphi d x d t=\int_{0}^{T} \int_{\mathbb{R}^{3}} \chi \varphi d x d t
\end{aligned}
$$

and from here by the arbitrariness of $\varphi$ we obtain $\tau_{\theta} \chi=\chi$ for a.e. $0<t<T$ and a.e. $x \in \mathbb{R}^{3}$ which proves the axisymmetry of $\chi$. 
For $\theta \in \mathbb{R}$ by the axisymmetry of $u_{\epsilon_{k}}$ we have $T_{\theta} u_{\epsilon_{k}}=u_{\epsilon_{k}}$. Thus for $\varphi \in\left(C_{c}\left(\mathbb{R}^{4}\right)\right)^{3}$ by the weak convergence (4.31) we have

$$
\begin{aligned}
\int_{0}^{T} \int_{\mathbb{R}^{3}}\left(T_{\theta} u\right)^{T} \varphi d x d t & =\int_{0}^{T} \int_{\mathbb{R}^{3}} u^{T}\left(T_{-\theta} \varphi\right) d x d t \\
& =\lim _{k \rightarrow \infty} \int_{0}^{T} \int_{\mathbb{R}^{3}} u_{\epsilon_{k}}^{T}\left(T_{-\theta} \varphi\right) d x d t=\lim _{k \rightarrow \infty} \int_{0}^{T} \int_{\mathbb{R}^{3}}\left(T_{\theta} u_{\epsilon_{k}}\right)^{T} \varphi d x d t \\
& =\lim _{k \rightarrow \infty} \int_{0}^{T} \int_{\mathbb{R}^{3}} u_{\epsilon_{k}}^{T} \varphi d x d t=\int_{0}^{T} \int_{\mathbb{R}^{3}} u^{T} \varphi d x d t
\end{aligned}
$$

and from here by the arbitrariness of $\varphi$ we obtain $T_{\theta} u=u$ for a.e. $0<t<T$ and a.e. $x \in \mathbb{R}^{3}$ which proves the axisymmetry of $u$.

Now let us prove the axisymmetry properties of $V$. Let $\theta \in \mathbb{R}$ and $\varphi \in C_{c}\left(\mathbb{R}^{7}\right)$ then using (3.1) we compute

$$
\begin{aligned}
\int_{0}^{T} & \int_{\mathbb{R}^{3} \times \mathbb{S}^{2}} \varphi(t, x, y)\left(V(t) \circ O^{T}(\theta)\right)(d(x, y)) d t \\
= & \int_{0}^{T} \int_{\mathbb{R}^{3} \times \mathbb{S}^{2}} \varphi(t, O(\theta) x, O(\theta) y) V(t)(d(x, y)) d t \\
= & \lim _{k \rightarrow \infty} \int_{0}^{T} \int_{\mathbb{R}^{3} \times \mathbb{S}^{2}} \varphi(t, O(\theta) x, O(\theta) y) V_{\epsilon_{k}}(t)(d(x, y)) d t \\
= & \lim _{k \rightarrow \infty} \int_{0}^{T} \int_{\mathbb{R}^{3} \cap\left\{\left|\nabla \chi_{\epsilon_{k}}\right| \neq 0\right\}} \varphi\left(t, O(\theta) x, O(\theta)\left(-\frac{\nabla \chi_{\epsilon_{k}}}{\left|\nabla \chi_{\epsilon_{k}}\right|}\right)\left|\nabla \chi_{\epsilon_{k}}(x)\right| d x d t\right. \\
= & \lim _{k \rightarrow \infty} \int_{0}^{T} \int_{\mathbb{R}^{3} \cap\left\{x \in \mathbb{R}^{3}|| \nabla \chi_{\epsilon_{k}}\left(t, O^{T}(\theta) x\right) \mid \neq 0\right\}} \varphi(t) \\
& \varphi\left(t, x, O(\theta)\left(-\frac{\nabla \chi_{\epsilon_{k}}\left(O^{T}(\theta) x\right)}{\left|\nabla \chi_{\epsilon_{k}}\left(O^{T}(\theta) x\right)\right|}\right)\left|\nabla \chi_{\epsilon_{k}}\left(O^{T}(\theta) x\right)\right| d x d t\right. \\
= & \left.\lim _{k \rightarrow \infty} \int_{0}^{T} \int_{\mathbb{R}^{3} \cap\left\{x \in \mathbb{R}^{3}|| \nabla \chi_{\epsilon_{k}}(t, x) \mid \neq 0\right\}} \varphi\left(t, x,-\frac{\nabla \chi_{\epsilon_{k}}(x)}{\left|\nabla \chi_{\epsilon_{k}}(x)\right|}\right)\right)\left|\nabla \chi_{\epsilon_{k}}(x)\right| d x d t \\
= & \lim _{k \rightarrow \infty} \int_{0}^{T} \int_{\mathbb{R}^{3} \times \mathbb{S}^{2}} \varphi(t, x, y) V_{\epsilon_{k}}(t)(d(x, y)) d t \\
= & \int_{0}^{T} \int_{\mathbb{R}^{3} \times \mathbb{S}^{2}} \varphi(t, x, y) V(t)(d(x, y)) d t
\end{aligned}
$$

and by the arbitrariness of $\varphi$ we obtain that for a.e. $0<t<T, V(t)$ satisfies (1.13).

It is easy to compute and see that

$$
\frac{d}{d \theta} O(\theta)=O\left(\theta+\frac{\pi}{2}\right) \Pi
$$

where

$$
\Pi=\left[\begin{array}{lr}
I_{2} & 0_{2} \\
0_{2}^{T} & 0
\end{array}\right],
$$

here $I_{2}$ is the $2 \times 2$ identity matrix.

By the axisymmetry of $\chi_{\epsilon}$ we can compute

$$
0=\frac{d}{d \theta} \chi_{\epsilon}(t, x)=\frac{d}{d \theta} \chi_{\epsilon}\left(t, O^{T}(\theta) x\right)=\nabla \chi_{\epsilon}\left(t, O^{T}(\theta) x\right)^{T} O\left(\theta+\frac{\pi}{2}\right) \Pi x
$$


and in particular for $\theta=0$ we obtain

$$
0=\nabla \chi_{\epsilon}(t, x)^{T} O\left(\frac{\pi}{2}\right) \Pi x \quad \text { for } 0<t<T \text { and } x \in \mathbb{R}^{3} .
$$

Let us define

$$
Q=\left\{(x, y) \in \mathbb{R}^{6} \mid y^{T} O\left(\frac{\pi}{2}\right) \Pi x=0\right\}
$$

then $Q$ is closed and is not equal to $\mathbb{R}^{6}$.

Let $\varphi \in C_{c}\left(\mathbb{R} \times Q^{c}\right)$ then

$$
\varphi=h(t, x, y) y^{T} O\left(\frac{\pi}{2}\right) \Pi x
$$

where

$$
h=\frac{\varphi}{y^{T} O\left(\frac{\pi}{2}\right) \Pi x} \in C_{c}\left(\mathbb{R}^{7}\right) .
$$

Now we may compute using (4.54)

$$
\begin{aligned}
& \int_{0}^{T} \int_{\mathbb{R}^{3} \times \mathbb{S}^{2}} \varphi V(t)(d(x, n)) d t=\lim _{k \rightarrow \infty} \int_{0}^{T} \int_{\mathbb{R}^{3} \times \mathbb{S}^{2}} \varphi V_{\epsilon_{k}}(t)(d(x, n)) d t \\
& =\lim _{k \rightarrow \infty} \int_{0}^{T} \int_{\mathbb{R}^{3} \cap\left\{\left|\nabla \chi_{\epsilon_{k}}\right| \neq 0\right\}} \varphi\left(t, x,-\frac{\nabla \chi_{\epsilon_{k}}}{\left|\nabla \chi_{\epsilon_{k}}\right|}\right)\left|\nabla \chi_{\epsilon_{k}}\right| d x d t \\
& =\lim _{k \rightarrow \infty} \int_{0}^{T} \int_{\mathbb{R}^{3} \cap\left\{\left|\nabla \chi_{\epsilon_{k}}\right| \neq 0\right\}} h\left(t, x,-\frac{\nabla \chi_{\epsilon_{k}}}{\left|\nabla \chi_{\epsilon_{k}}\right|}\right)\left(-\frac{\nabla \chi_{\epsilon_{k}}}{\left|\nabla \chi_{\epsilon_{k}}\right|}\right)^{T} O\left(\frac{\pi}{2}\right) \Pi x\left|\nabla \chi_{\epsilon_{k}}\right| d x d t \\
& =-\lim _{k \rightarrow \infty} \int_{0}^{T} \int_{\mathbb{R}^{3} \cap\left\{\left|\nabla \chi_{\epsilon_{k}}\right| \neq 0\right\}} h\left(t, x,-\frac{\nabla \chi_{\epsilon_{k}}}{\left|\nabla \chi_{\epsilon_{k}}\right|}\right) \nabla \chi_{\epsilon_{k}}^{T} O\left(\frac{\pi}{2}\right) \Pi x d x d t=0 .
\end{aligned}
$$

Now by (4.55) and the arbitrariness of $\varphi \in C_{c}\left(\mathbb{R} \times Q^{c}\right)$ we obtain that for a.e. $0<t<T, V(t)$ satisfies (1.14).

Acknowledgments. The research leading to these results has received funding from Lithuanian-Swiss cooperation programme to reduce economic and social disparities within the enlarged European Union under Project Agreement No. CH-3-SMM-01/0. The author would also like to thank the Isaac Newton Institute for Mathematical Sciences, Cambridge, for support and hospitality during the programme Free Boundary Problems and Related Topics, where partially the work on this paper was undertaken.

\section{References}

[1] Abels, H.: Initial-value problem for the Navier-Stokes equations with a free surface in Lq-Sobolev spaces. Adv. Differ. Equ. 10(1), 45-64 (2005)

[2] Abels, H.: On generalized solutions of two-phase flows for viscous incompressible fluids. Interfaces Free Bound. 9(1), 31$65(2007)$

[3] Beale, J.T.: The initial value problem for the Navier-Stokes equations with a free surface. Commun. Pure Appl. Math. 34(3), 359-392 (1981)

[4] Beale, J.T.: Large-time regularity of viscous surface waves. Arch. Ration. Mech. Anal. 84(4),307-352 (1983/1984)

[5] Diestel, J., Uhl, J.J. Jr.: Vector measures. In: Mathematical Surveys, No. 15, pp. xiii + 322. American Mathematical Society, Providence (1977)

[6] DiPerna, R.J., Lions, P.L.: Ordinary differential equations, transport theory and Sobolev spaces. Invent. Math. 98(3), 511-547 (1989)

[7] Evans, L.C.: Partial differential equations. In: Graduate Studies in Mathematics, 2nd edn, vol. 19, pp. xxii + 749. American Mathematical Society, Providence (2010)

[8] Pileckas, K., Solonnikov, V.A.: Viscous incompressible free-surface flow down an inclined perturbed plane. Ann. Univ. Ferrara Sez. VII Sci. Mat. 60(1), 225-244 (2014) 
[9] Plotnikov, P.I.: Generalized solutions of a problem on the motion of a non-Newtonian fluid with a free boundary. Sibirsk. Mat. Zh. 34(4), 127-141 (1993). [iii, ix; translation in Sib. Math. J. 34(4), 704-716 (1993)]

[10] Roubícěk, T.: A generalization of the Lions-Temam compact imbedding theorem. Casopis Pěst. Mat. 115(4), 338-342 (1990)

[11] Simon, L.: Lectures on geometric measure theory. In: Proceedings of the Centre for Mathematical Analysis, vol. 3, pp. vii+272. Australian National University, Centre for Mathematical Analysis, Canberra (1983)

[12] Shibata, Y., Shimizu, S.: Lp-Lq maximal regularity and viscous incompressible flows with free surface. Proc. Jpn. Acad. Ser. A Math. Sci. 81(9), 151-155 (2005)

[13] Solonnikov, V.A.: Estimates of the solution of a certain initial-boundary value problem for a linear nonstationary system of Navier-Stokes equations. (Russian. English summary) Boundary value problems of mathematical physics and related questions in the theory of functions, 9. Zap. Naučn. Sem. Leningrad. Otdel Mat. Inst. Steklov. (LOMI) 59, 178-254, 257 (1976)

[14] Solonnikov, V.A.: Unsteady motion of an isolated volume of a viscous incompressible fluid (Russian). Izv. Akad. Nauk SSSR Ser. Mat. 51(5), 1065-1087, 1118 (1987). [translation in Math. USSR-Izv. 31(2), 381-405 (1988)]

[15] Stein, E.: Harmonic analysis: real-variable methods, orthogonality, and oscillatory integrals. In: Princeton Mathematical Series, 43, Monographs in Harmonic Analysis, vol. III, pp. xiv + 695. Princeton University, Princeton (1993)

[16] Stein, E.M., Weiss, G.: Introduction to Fourier analysis on Euclidean spaces. In: Princeton Mathematical Series, No. 32, pp. $\mathrm{x}+$ 297. Princeton University, Princeton (1971)

[17] Tani, A., Tanaka, N.: Large-time existence of surface waves in incompressible viscous fluids with or without surface tension. Arch. Ration. Mech. Anal. 130(4), 303-314 (1995)

Karen Yeressian

Institute of Mathematics

University of Zurich

Winterthurerstrasse 190

8057 Zurich

Switzerland

e-mail: karen.yeressian@math.uzh.ch

(accepted: April 19, 2015; published online: June 25, 2015) 